\title{
Backward Ornstein-Uhlenbeck Transition Operators and Mild Solutions of Non-Autonomous Hamilton-Jacobi Equations in Banach Spaces
}

\author{
Rafael Serrano ${ }^{1}$ \\ ${ }^{1}$ Universidad del Rosario, Bogotá, Colombia \\ Correspondence: Rafael Serrano, Universidad del Rosario, Calle 12c No. 4-69, Bogotá, Colombia. E-mail: \\ rafael.serrano@urosario.edu.co
}

Received: August 19, 2014 Accepted: October 9, 2014 Online Published: October 20, 2014

doi:10.5539/jmr.v6n4p55 URL: http://dx.doi.org/10.5539/jmr.v6n4p55

\begin{abstract}
In this paper we revisit the mild-solution approach to second-order semi-linear PDEs of Hamilton-Jacobi type in infinite-dimensional spaces. We show that a well-known result on existence of mild solutions in Hilbert spaces can be easily extended to non-autonomous Hamilton-Jacobi equations in Banach spaces. The main tool is the regularizing property of Ornstein-Uhlenbeck transition evolution operators for stochastic Cauchy problems in Banach spaces with time-dependent coefficients.
\end{abstract}

Keywords: Ornstein-Uhlenbeck processes, infinite-dimensional Hamilton-Jacobi equations, mild solution, regularizing property, Banach spaces

\section{Introduction}

Let $\mathbf{E}$ be a real Banach space and let $T>0$ be fixed. The object of this paper is to study the existence of a mild solution $V:[0, T] \times \mathbf{E} \rightarrow \mathbb{R}$ to the following final-value problem for the non-autonomous semi-linear HamiltonJacobi partial differential equation $(\mathrm{HJ}-\mathrm{PDE})$ on $[0, T] \times \mathbf{E}$,

$$
\begin{aligned}
\frac{\partial V}{\partial t}(t, x)+L_{t} V(t, \cdot)(x)+\mathcal{H}\left(t, x, D_{x} V(t, x)\right) & =0, \quad(t, x) \in[0, T] \times \mathbf{E} \\
V(T, x) & =\varphi(x) .
\end{aligned}
$$

The final condition $\varphi: \mathbf{E} \rightarrow \mathbb{R}$ and the nonlinear Hamiltonian operator $\mathcal{H}:[0, T] \times \mathbf{E} \times \mathbf{E}^{*} \rightarrow \mathbb{R}$ are given, and for each $t \in[0, T], L_{t}$ is the second-order differential operator

$$
\left(L_{t} \phi\right)(x):=\left\langle-A(t) x, D_{x} \phi(x)\right\rangle+\frac{1}{2} \operatorname{Tr}_{\mathbf{H}}\left[G(t)^{*} D_{x}^{2} \phi(x) G(t)\right], x \in D(A(t)) .
$$

Here $\langle\cdot, \cdot\rangle$ denotes the duality pairing between $\mathbf{E}$ and its dual $\mathbf{E}^{*},\{-A(t)\}_{t \in[0, T]}$ are densely defined closed linear operators generating a parabolic evolution family on $\mathbf{E},\{G(t)\}_{t \in[0, T]}$ are (possibly unbounded) linear operators from a Hilbert space $\mathbf{H}$ into $\mathbf{E}$, $\operatorname{Tr}_{\mathbf{H}}[\cdot]$ denotes the trace in $\mathbf{H}$, and $D_{x} \phi(x), D_{x}^{2} \phi(x)$ denote first and second order Fréchet derivatives of $\phi: \mathbf{E} \rightarrow \mathbb{R}$ at $x \in D(A(t))$.

In this paper we revisit the mild solution approach to Hamilton-Jacobi equations initiated by Da Prato (1985) and Cannarsa and Da Prato (1991), and continued by Gozzi (1995, 1996), Cerrai (2001a, 2001b) and Masiero (2005) (see also Da Prato \& Zabczyk, 2002; Zabczyk, 1999 and the references therein). This approach consists in rewriting Equation (1.1) in mild-integral form (cf. variation-of-constants formula)

$$
V(t, x)=[P(t, T) \varphi](x)+\int_{t}^{T}\left[P(t, r) \mathcal{H}\left(r, \cdot, D_{x} V(r, \cdot)\right)\right](x) d r, \quad(t, x) \in[0, T] \times \mathbf{E}
$$

where $P(s, t)$ is the backward transition evolution operator

$$
[P(s, t) \varphi](x):=\mathbb{E}[\varphi(Z(t)) \mid Z(s)=x], x \in \mathbf{E}, t \in[s, T], \varphi \in \mathcal{B}_{b}(\mathbf{E})
$$


associated with the Ornstein-Uhlenbeck process $\{Z(t)\}_{t \in[0, T]}$ solution to the non-autonomous stochastic Cauchy problem on $\mathbf{E}$

$$
d Z(t)+A(t) Z(t) d t=G(t) d W(t), \quad t \in[0, T] .
$$

Here $\{W(t)\}_{t \in[0, T]}$ is an $\mathbf{H}$-cylindrical Wiener process defined on a probability space $(\Omega, \mathcal{F}, \mathbb{P}), \mathbb{E}[\cdot]$ denotes expectation in the Bochner-integral sense with respect to the probability measure $\mathbb{P}$ and $\mathcal{B}_{b}(\mathbf{E})$ denotes the set of bounded Borel-measurable real-valued maps on $\mathbf{E}$.

Under the so-called null-controllability condition (see Assumption A.2 in Section 5 below) the backward transition operators $P(s, t)$ satisfy a strong regularizing property, see Theorem 5.8. For the case in which $\mathbf{E}$ is a Hilbert space and Equation (1.1) is autonomous with respect to time variable (i.e. $A(t)$ and $G(t)$ do not depend on $t$ ), this regularizing property has been used in conjunction with a fixed point argument to prove existence of a unique solution to the integral Equation (1.2) in a certain space of functions, see e.g. Theorem 9.3 in Zabczyk (1999, Sec. 9), Da Prato and Zabczyk (2002, Part III) and Masiero (2005).

The main purpose of this paper is to show that this result can be easily generalized to the non-autonomous and Banach-space setting. Namely, we obtain the following (see Theorem 6.6 below)

Theorem Let $\varphi \in C_{b}(\mathbf{E})$. Suppose Assumptions (AT) and A.1-A.4 hold true. Then there exists an unique mild solution to Equation (1.1).

We refer the reader to Sections 4-6 below for the precise statement of Assumptions (AT) and A.1-A.4. As an example, we consider a non-autonomous $\mathrm{HJ}$ equation in $L^{p}(0,1)$ with $p \geq 2$, see Example 6.7 below.

It should be emphasized that our proof does not present any significant innovation as we follow closely the arguments in the proof for the Hilbert-space case in (Masiero 2005, Theorem 2.9). However, to the best of our knowledge, this is the first paper that deals with infinite-dimensional non-autonomous semi-linear HJ equations in the general Banach-space framework, particularly in Lesbesgue spaces $L^{p}(O)$ with $p \geq 2$. This is our main motivation to study HJ equations in a more general Banach-space setting that led to the writing of this paper.

The rest of the paper is organized as follows. In section 2 we recall some basic facts on Gaussian measures in Banach spaces, reproducing kernel Hilbert spaces and the Cameron-Martin formula. We present an alternative proof of a well-known result on regularizing property of Gaussian convolutions which first appeared in the seminal paper Gross (1967). In section 3 we review some results from van Neerven and Weis (2005a) on stochastic integration of deterministic operator valued functions with respect to a cylindrical Wiener process.

In section 4 we recall the setting of Acquistapace and Terreni for parabolic evolution families and non-autonomous evolution equations. In section 5 we introduce backward Ornstein-Uhlenbeck (OU) transition evolution operators in Banach spaces and extend some results from (van Neerven 1998, Section 1) on the relation between the associated reproducing Kernel Hilbert spaces. In section 6, we state and prove the final result Theorem 6.6. Throughout, as the main working example, we consider a linear parabolic second-order stochastic PDE with time-dependent coefficients and space-time white noise formulated as an evolution equation in $L^{p}(0,1)$ with $p \geq 2$. We prove the transition operators of the (mild) solution verify the assumptions of the main result. This leads to our final Example 6.7 .

Discussion. Of particular interest are Hamiltonians $\mathcal{H}$ of the form

$$
\mathcal{H}(t, x, p)=\inf _{u \in M}\{\langle F(t, x, u), p\rangle+l(t, x, u)\}, \quad(t, x, p) \in[0, T] \times \mathbf{E} \times \mathbf{E}^{*}
$$

where $M$ is a separable metric space, $F:[0, T] \times \mathbf{E} \times M \rightarrow \mathbf{E}$ and $l:[0, T] \times \mathbf{E} \times M \rightarrow(-\infty, \infty]$. In this case, Equation (1.1) is the Hamilton-Jacobi-Bellman PDE associated with the dynamic programming principle approach to the following finite-horizon stochastic optimal control problem

$$
\operatorname{minimize} \quad J(U)=\mathbb{E}\left[\int_{0}^{T} l\left(t, X^{U}(t), U(t)\right) d t+\Psi(X(T))\right]
$$

where $U=\{U(t)\}_{t \in[0, T]}$ is an $M$-valued control process and $\left\{X^{U}(t)\right\}_{t \in[0, T]}$ is the $\mathbf{E}$-valued solution to the controlled non-autonomous stochastic evolution equation with additive noise

$$
\begin{aligned}
d X(t)+A(t) X(t) d t & =F(t, X(t), U(t)) d t+G(t) d W(t), \\
X(0) & =x_{0} \in \mathbf{E} .
\end{aligned}
$$


For the case in which $\mathbf{E}$ is Hilbert, under certain additional differentiability assumptions on the Hamiltonian (1.4), the mild solution of (1.1) can be used to formulate optimality criteria and verification-type results for optimal control problems in Hilbert spaces for stochastic PDEs, see e.g. (Da Prato \& Zabczyk, 2002, Part III) or (Masiero, 2005, Sec. 4-6). This can also be combined with Malliavin Calculus and backward stochastic evolution systems in Hilbert spaces to prove existence of an optimal feedback control, see e.g. Fuhrman and Tessitore (2002a, 2002b, 2004a, 2004b) and the references therein.

Using regularizing properties of stochastic convolutions, Masiero (2008) proved existence of mild solutions of a certain class of autonomous HJB equations on the space of continuous functions $C(O)$. Under additional, somewhat restrictive conditions on the nonlinear coefficient $F$, particularly a dissipative-type condition and a very specific form of dependence with respect to the control variable, Masiero also solved the control problem using backward SDEs but with no use of Malliavin calculus.

At the moment, we are unable to obtain optimality criteria and verification-type results for optimal control problems in Banach spaces for non-autonomous stochastic PDEs as this requires approximation results in $C_{b}(\mathbf{E})$ by smooth functions that do not seem available at the moment in the general Banach-space setting. However, we believe this can be overcome by employing recent results on Malliavin calculus in Banach spaces (see e.g. Maas, 2010). We will address this issue in a forthcoming paper.

\section{Gaussian Measures in Banach Spaces, Cameron-Martin Formula and Regularizing Property}

We recall first some basic facts on Gaussian measures in Banach spaces, particularly the Cameron-Martin formula and the smoothing property of Gaussian convolutions.

Let $\mathcal{B}(\mathbf{E})$ denote the Borel $\sigma$-algebra on the real Banach space $\mathbf{E}$, let $\mathbf{E}^{*}$ be the continuous dual of $\mathbf{E}$ and let $\langle\cdot, \cdot\rangle$ denote the duality pairing between $\mathbf{E}$ and $\mathbf{E}^{*}$.

Definition 2.1 A Radon measure $\mu$ on $(\mathbf{E}, \mathcal{B}(\mathbf{E}))$ is called Gaussian (resp. centered Gaussian) if, for any linear functional $x^{*} \in \mathbf{E}^{*}$, the image measure $\mu \circ\left\langle x^{*}, \cdot\right\rangle^{-1}$ is a Gaussian (resp. centered Gaussian) measure on $\mathbb{R}$.

If $\mu$ is a centered Gaussian measure on $\mathbf{E}$, there exists an unique bounded linear operator $\mathbf{C} \in \mathcal{L}\left(\mathbf{E}^{*}, \mathbf{E}\right)$ called the covariance operator of $\mu$, such that for all $x^{*}, y^{*} \in \mathbf{E}^{*}$ we have

$$
\left\langle\mathbf{C} x^{*}, y^{*}\right\rangle=\int_{\mathbf{E}}\left\langle x, x^{*}\right\rangle\left\langle x, y^{*}\right\rangle \mu(d x) .
$$

see e.g. Bogachev (1998). Notice that $C$ is positive in the sense that $\left\langle\mathbf{C} x^{*}, x^{*}\right\rangle \geq 0, \forall x^{*} \in \mathbf{E}^{*}$, and symmetric in the sense that $\left\langle\mathbf{C} x^{*}, y^{*}\right\rangle=\left\langle\mathbf{C} y^{*}, x^{*}\right\rangle, \forall x^{*}, y^{*} \in \mathbf{E}^{*}$. The Fourier transform $\hat{\mu}$ of $\mu$ is defined as

$$
\hat{\mu}\left(x^{*}\right)=\exp \left(-\frac{1}{2}\left\langle\mathbf{C} x^{*}, x^{*}\right\rangle\right), \quad x^{*} \in \mathbf{E}^{*} .
$$

This identity implies that two centered Gaussian measures are equal whenever their covariance operators are equal.

For any $\mathbf{C} \in \mathcal{L}\left(\mathbf{E}^{*}, \mathbf{E}\right)$ positive and symmetric, the bilinear form on $\operatorname{Im} C \subset \mathbf{E}$

$$
\left[\mathbf{C} x^{*}, \mathbf{C} y^{*}\right]:=\left\langle\mathbf{C} x^{*}, y^{*}\right\rangle, \quad x^{*}, y^{*} \in \mathbf{E}^{*}
$$

is a well-defined inner product. We denote with $H_{\mathbf{C}}$ the Hilbert space completion of $\operatorname{Im} \mathbf{C}$ with respect to this inner product. The inclusion mapping from $\operatorname{Im} \mathbf{C}$ into $\mathbf{E}$ is continuous with respect to the inner product $[\cdot, \cdot]_{H_{\mathbf{C}}}$ and extends uniquely to a bounded linear injection $i_{\mathbf{C}}: H_{\mathbf{C}} \hookrightarrow \mathbf{E}$.

Definition 2.2 The pair $\left(i_{\mathbf{C}}, H_{\mathbf{C}}\right)$ is called the reproducing kernel Hilbert space (RKHS) associated with $C$.

It can be easily shown that the adjoint operator $i_{\mathbf{C}}^{*}: \mathbf{E}^{*} \rightarrow H_{\mathbf{C}}$ satisfies $i_{\mathbf{C}}^{*} x^{*}=C x^{*}$ for all $x^{*} \in \mathbf{E}^{*}$. Therefore, $C$ admits the factorization

$$
C=i_{\mathbf{C}} \circ i_{\mathbf{C}}^{*}
$$

This factorization immediately implies that $C$ is weak*-to-weakly continuous and that, if $\mathbf{E}$ is separable, so is $H_{\mathbf{C}}$. We identify for the sake of simplicity $H_{\mathbf{C}}$ with its image $\operatorname{Im} i_{\mathbf{C}} \subset \mathbf{E}$.

Proposition 2.3 (van Neerven, 1998, Proposition 1.1) Let $\mathbf{C}, \tilde{\mathbf{C}} \in \mathcal{L}\left(\mathbf{E}^{*}, \mathbf{E}\right)$ be two positive symmetric operators. Then, for the corresponding reproducing kernel Hilbert spaces we have $H_{\mathbf{C}} \subset H_{\tilde{\mathbf{C}}}$ (as subsets of $\mathbf{E}$ ) if and only if there exist a constant $K>0$ such that

$$
\left\langle\mathbf{C} x^{*}, x^{*}\right\rangle \leq K\left\langle\tilde{\mathbf{C}} x^{*}, x^{*}\right\rangle, \quad \forall x^{*} \in \mathbf{E}^{*} .
$$


We will denote with $H_{\mu}$ (resp. $i_{\mu}$ ) instead of $H_{\mathbf{C}}$ (resp. $i_{\mathbf{C}}$ ) whenever $\mathbf{C}$ is the covariance operator of a Gaussian measure $\mu$ on $\mathbf{E}$. In this case, we introduce a linear isometry from $H_{\mu}$ into $L^{2}(\mathbf{E}, \mu)$ as follows: first observe that $\left\langle x^{*}, \cdot\right\rangle \in L^{2}(\mathbf{E}, \mu)$ for every linear functional $x^{*} \in \mathbf{E}^{*}$ and that we have

$$
\mathbb{E}^{\mu}\left|\left\langle x^{*}, \cdot\right\rangle\right|^{2}=\int_{\mathbf{E}}\left|\left\langle x, x^{*}\right\rangle\right|^{2} \mu(d x)=\left\langle\mathbf{C} x^{*}, x^{*}\right\rangle, \quad x^{*} \in \mathbf{E}^{*} .
$$

Here $\mathbb{E}^{\mu}$ denotes the expectation on the probability space $(\mathbf{E}, \mathcal{B}(\mathbf{E}), \mu)$. Since $\mathbf{C}$ is injective as an operator from $\mathbf{E}^{*}$ into $\operatorname{Im} \mathbf{C}$, the linear map

$$
\operatorname{Im} \mathbf{C} \ni \mathbf{C}\left(x^{*}\right) \mapsto\left\langle x^{*}, \cdot\right\rangle \in L^{2}(\mathbf{E}, \mu)
$$

is well-defined and is an isometry in view of (2.1). We denote by

$$
\phi_{\mu}: H_{\mu} \rightarrow L^{2}(\mathbf{E}, \mu)
$$

the unique extension of the isometry (2.2) to $H_{\mu}$. This isometry, known as the Paley-Wiener integral, has the property that for each $h \in H_{\mu}, \phi_{\mu}(h)$ is a $\mathcal{N}\left(0,|h|_{H_{\mu}}^{2}\right)$ random variable. Indeed, for $h \in H_{\mu}$ fixed, if $\left(x_{n}^{*}\right)_{n}$ is a sequence in $\mathbf{E}^{*}$ such that $\mathbf{C} x_{n}^{*} \rightarrow h$ in $H_{\mu}$, then

$$
\left\langle x_{n}^{*}, \cdot\right\rangle=\phi_{\mu}\left(\mathbf{C} x_{n}^{*}\right) \rightarrow \phi_{\mu}(h), \quad \text { in } L^{2}(\mathbf{E}, \mu)
$$

and this implies, in particular, that $\mathbb{E}^{\mu}\left[e^{i \lambda\left\langle x_{n}^{*},\right\rangle}\right] \rightarrow \mathbb{E}^{\mu}\left[e^{i \lambda \phi_{\mu}(h)}\right]$ as $n \rightarrow \infty$ for all $\lambda \in \mathbb{R}$. Since $\left\langle x_{n}^{*}, \cdot\right\rangle$ is normally distributed with mean 0 and variance $\left|\mathbf{C} x_{n}^{*}\right|_{H_{\mu}}^{2}$, we have

$$
\mathbb{E}^{\mu}\left[e^{i \lambda\left\langle x_{n}^{*},\right\rangle}\right]=\exp \left(-\frac{\lambda^{2}}{2}\left|\mathbf{C} x_{n}^{*}\right|_{H_{\mu}}^{2}\right), \quad \lambda \in \mathbb{R},
$$

and by dominated convergence, taking the limit as $n \rightarrow \infty$ we get

$$
\mathbb{E}^{\mu}\left[e^{i \lambda \phi_{\mu}(h)}\right]=\exp \left(-\frac{\lambda^{2}}{2}|h|_{H_{\mu}}^{2}\right), \quad \lambda \in \mathbb{R},
$$

which implies that $\phi_{\mu}(h)$ is a $\mathcal{N}\left(0,|h|_{H_{\mu}}^{2}\right)$-distributed random variable.

Definition 2.4 For each $h \in H_{\mu}$ we denote by $\mu^{h}$ the image of the measure $\mu$ under the translation $z \mapsto z+h$, that is,

$$
\mu^{h}(A):=\mu(A-h), \quad A \in \mathcal{B}(\mathbf{E}) .
$$

We call $\mu^{h}$ the shift of the measure $\mu$ by the vector $h$.

Theorem 2.5 (Cameron-Martin formula) Let $\mu$ be a centered Gaussian measure on $\mathbf{E}$ with covariance operator $\mathbf{C} \in \mathcal{L}\left(\mathbf{E}^{*}, \mathbf{E}\right)$ and let $\left(i_{\mu}, H_{\mu}\right)$ denote the RKHS associated with $\mu$. Then, for any $h \in H_{\mu}$, the measure $\mu^{h}$ is absolutely continuous with respect to $\mu$ and we have

$$
\frac{d \mu^{h}}{d \mu}=\rho_{h}, \mu-a . s .
$$

with $\rho_{h}:=\exp \left(\phi_{\mu}(h)-\frac{1}{2}|h|_{H_{\mu}}^{2}\right), h \in H_{\mu}$.

Proof. See (Bogachev 1998, Corollary 2.4.3).

For the remainder of this section, we fix $\varphi \in \mathcal{B}_{b}(\mathbf{E})$ and define the mapping $\psi: \mathbf{E} \rightarrow \mathbb{R}$ as

$$
\psi(x):=\int_{\mathbf{E}} \varphi(x+z) \mu(d z), \quad x \in \mathbf{E} .
$$

Recall that $\psi: \mathbf{E} \rightarrow \mathbb{R}$ is Fréchet differentiable at $x \in \mathbf{E}$ in the direction of $H_{\mu}$ if there exists an element of $H_{\mu}^{*}$, denoted by $D_{H_{\mu}} \psi(x)$, such that

$$
\lim _{\substack{y \in H_{\mu} \\ y \rightarrow 0}} \frac{\left|\psi(x+y)-\psi(x)-\left(D_{H_{\mu}} \psi(x)\right)(y)\right|}{|y|_{H_{\mu}}}=0 .
$$


The following regularizing property is a classical result proved by L. Gross in his seminal paper (Gross 1967, Proposition 9) using directly the notion of Fréchet derivative. Here we present an alternative proof based on Gâteaux differentiability.

Proposition 2.6 The map $\psi: \mathbf{E} \rightarrow \mathbb{R}$ is infinitely Fréchet differentiable in the direction of $H_{\mu}$. The first Fréchet derivative of $\psi$ at $x \in \mathbf{E}$ in the direction of $y \in H_{\mu}$ is given by

$$
\left(D_{H_{\mu}} \psi(x)\right)(y)=\int_{\mathbf{E}} \varphi(x+z) \phi_{\mu}(y)(z) \mu(d z)
$$

and the second Fréchet derivative of $\psi$ at $x \in \mathbf{E}$ in the directions $y_{1}, y_{2} \in H_{\mu}$ is given by

$$
\left(D_{H_{\mu}}^{2} \psi(x)\right)\left(y_{1}, y_{2}\right)=-\psi(x)\left[y_{1}, y_{2}\right]_{H_{\mu}}+\int_{\mathbf{E}} \varphi(x+z) \phi_{\mu}\left(y_{1}\right)(z) \phi_{\mu}\left(y_{2}\right)(z) \mu(d z) .
$$

Moreover we have the estimates $\left\|D_{H_{\mu}} \psi(x)\right\|_{H_{\mu}^{*}} \leq|\varphi|_{0}$ and $\left\|D_{H_{\mu}}^{2} \psi(x)\right\|_{\mathcal{L}\left(H_{\mu}, H_{\mu}^{*}\right)} \leq 2|\varphi|_{0}$.

Proof. Let us prove first that $\psi$ is Gâteaux differentiable in the direction of $H_{\mu}$, i.e. that for all $x \in \mathbf{E}$ and $y \in H_{\mu}$, the mapping

$$
\mathbb{R} \ni \alpha \mapsto \psi(x+\alpha y) \in \mathbb{R}
$$

is differentiable at $\alpha=0$. Let $x \in \mathbf{E}$ and $y \in H_{\mu}$ be fixed and let $\alpha \in \mathbb{R}$. Observe that by the Cameron-Martin formula, we have

$$
\psi(x+\alpha y)=\int_{\mathbf{E}} \varphi(x+z) \mu^{\alpha y}(d z)=\int_{\mathbf{E}} \varphi(x+z) \rho_{\alpha y}(z) \mu(d z) .
$$

Since $\phi_{\mu}(\alpha y)=\alpha \phi_{\mu}(y)$ in $L^{2}(\mathbf{E}, \mu)$ observe that the random variable $\rho_{\alpha y}=\exp \left(\alpha \phi_{\mu}(y)-\frac{1}{2}|\alpha y|_{H_{\mu}}^{2}\right)$ is defined on a set $\widehat{\mathbf{E}}=\widehat{\mathbf{E}}(y)$ of full $\mu$-measure which depends only on $y$, for all $\alpha \in \mathbb{R}$. Thus, the mapping

$$
g: \mathbb{R} \times \mathbf{E} \ni(\alpha, z) \mapsto g(\alpha, z):=\rho_{\alpha y}(z) \in \mathbb{R}
$$

is well-defined and measurable. Moreover, for $\varepsilon>0$ fixed we have the following estimate for all $\left|\alpha_{0}\right|<\varepsilon, z \in \widehat{\mathbf{E}}$,

$$
\left|\frac{\partial g}{\partial \alpha}\left(\alpha_{0}, z\right)\right|=\left.\rho\left(\alpha_{0} y, z\right)\left|\phi_{\mu}(y)(z)-\alpha_{0}\right| y\right|_{H_{\mu}} ^{2} \mid \leq \exp \left(\varepsilon\left|\phi_{\mu}(y)(z)\right|\right)\left(\left|\phi_{\mu}(y)(z)\right|+\varepsilon|y|_{H_{\mu}}^{2}\right) .
$$

We know $\phi_{\mu}(y)$ is Gaussian random variable with moment generating function

$$
\mathbb{E}^{\mu}\left[e^{\lambda \phi_{\mu}(y)}\right]=\exp \left(\frac{\lambda^{2}}{2}|y|_{H_{\mu}}^{2}\right), \quad \lambda \in \mathbb{R} .
$$

This implies, in particular, that $\exp \left(\varepsilon\left|\phi_{\mu}(y)\right|\right)$ belongs to $L^{2}(\mathbf{E}, \mu)$. Since $\phi_{\mu}(y) \in L^{2}(\mathbf{E}, \mu)$, by Hölder's inequality the right hand side in (2.8) belongs to $L^{1}(\mathbf{E}, \mu)$. Thus we may differentiate in the right hand-side of (2.6) with respect to $\alpha$ under the sign and obtain that the Gâteaux derivative of $\psi$ at $x$ in the direction of $y$ is given by

$$
\left(d_{H_{\mu}} \psi(x)\right)(y)=\left.\frac{d}{d \alpha}\right|_{\alpha=0} \psi(x+\alpha y)=\int_{\mathbf{E}} \varphi(x+z)\left[\left.\frac{\partial}{\partial \alpha}\right|_{\alpha=0} \rho_{\alpha y}(z)\right] \mu(d z),=\int_{\mathbf{E}} \varphi(x+z) \phi_{\mu}(y)(z) \mu(d z),
$$

as well as the following estimate

$$
\left\|d_{H_{\mu}} \psi(x)\right\|_{\mathcal{L}\left(H_{\mu}, \mathbb{R}\right)} \leq|\varphi|_{0} .
$$

In turn this implies that the Gateaux derivative $d \psi: H_{\mu} \rightarrow \mathcal{L}\left(H_{\mu}, \mathbb{R}\right)$ is continuous and uniformly bounded. Since $\psi$ is also continuous and uniformly bounded on $H_{\mu}$, by Theorem 3 in (Aronszajn 1976, Ch. 2, Section 1) we conclude that $\psi$ is Fréchet differentiable in the direction of $H_{\mu}$ and (2.4) follows.

For the second-order Gâteaux derivative, if $y_{1}, y_{2} \in H_{\mu}$ and $\alpha \in \mathbb{R}$ we have

$$
\begin{aligned}
\left(d_{H_{\mu}} \psi\left(x+\alpha y_{2}\right)\right)\left(y_{1}\right) & =\int_{\mathbf{E}} \varphi\left(x+\alpha y_{2}+z\right) \phi_{\mu}\left(y_{1}\right)(z) \mu(d z) \\
& =\int_{\mathbf{E}} \varphi(x+\xi) \phi_{\mu}\left(y_{1}\right)\left(\xi-\alpha y_{2}\right) \mu^{\alpha y_{2}}(d \xi) \\
& =\int_{\mathbf{E}} \varphi(x+\xi) \phi_{\mu}\left(y_{1}\right)\left(\xi-\alpha y_{2}\right) \rho_{\alpha y_{2}}(\xi) \mu(d \xi)
\end{aligned}
$$


where we have used again the Cameron-Martin formula and the change of variable $\xi=z+\alpha y_{2}$ whose push-forward measure with respect with $\mu$ is given by $\mu^{\alpha y_{2}}$.

If $y_{1}=\mathbf{C} x_{1}^{*}$ for some $x_{1}^{*} \in \mathbf{E}^{*}$, from the definition of $\phi_{\mu}$ it follows that

$$
\phi_{\mu}\left(y_{1}\right)\left(\xi-\alpha y_{2}\right)=\left\langle x_{1}^{*}, \xi-\alpha y_{2}\right\rangle=\left\langle x_{1}^{*}, \xi\right\rangle-\alpha\left\langle x_{1}^{*}, y_{2}\right\rangle=\phi_{\mu}\left(y_{1}\right)(\xi)-\alpha\left[y_{1}, y_{2}\right]_{H_{\mu}}
$$

in which case we have

$$
\left(d_{H_{\mu}} \psi\left(x+\alpha y_{2}\right)\right)\left(y_{1}\right)=\int_{\mathbf{E}} \varphi(x+\xi)\left(\phi_{\mu}\left(y_{1}\right)(\xi)-\alpha\left[y_{1}, y_{2}\right]_{H_{\mu}}\right) \rho_{\alpha y_{2}}(\xi) \mu(d \xi) .
$$

Since both sides of (2.9) are continuous in $y_{1} \in H_{\mu}$ and $\operatorname{Im} \mathbf{C}$ is dense in $H_{\mu}$, the above equality holds for any $y_{1} \in H_{\mu}$. In addition, the equality

$$
\left.\frac{\partial}{\partial \alpha}\right|_{\alpha=0}\left[\left(\phi_{\mu}\left(y_{1}\right)(\xi)-\alpha\left[y_{1}, y_{2}\right]_{H_{\mu}}\right) \rho\left(\alpha y_{2}, \xi\right)\right]=-\left[y_{1}, y_{2}\right]_{H_{\mu}}+\phi_{\mu}\left(y_{1}\right)(\xi) \phi_{\mu}\left(y_{2}\right)(\xi),
$$

holds for all $\xi$ in a subset of $\mathbf{E}$ with full $\mu$-measure that only depends on $y_{2}$. Again, we can differentiate under the integral sign with respect to $\alpha$ to obtain the second Gâteaux derivative of $\psi$ at $x$ in the direction o $y_{1}$ and $y_{2}$,

$$
\begin{aligned}
\left(d_{H_{\mu}}^{2} \psi(x)\right)\left(y_{1}, y_{2}\right) & =\left.\frac{d}{d \alpha}\right|_{\alpha=0}\left(d_{H_{\mu}} \psi\left(x+\alpha y_{2}\right)\right)\left(y_{1}\right) \\
& =\left.\int_{\mathbf{E}} \varphi(x+\xi) \frac{\partial}{\partial \alpha}\right|_{\alpha=0}\left[\left(\phi_{\mu}\left(y_{1}\right)(\xi)-\alpha\left[y_{1}, y_{2}\right]_{H_{\mu}}\right) \rho_{\alpha y_{2}}(\xi)\right] \mu(d \xi) \\
& =\int_{\mathbf{E}} \varphi(x+\xi)\left(\phi_{\mu}\left(y_{1}\right)(\xi) \phi_{\mu}\left(y_{2}\right)(\xi)-\left[y_{1}, y_{2}\right]_{H_{\mu}}\right) \mu(d \xi)
\end{aligned}
$$

together with the following estimate

$$
\left\|d_{H_{\mu}}^{2} \psi(x)\right\|_{\mathcal{L}\left(H_{\mu}, H_{\mu}^{*}\right)} \leq 2|\varphi|_{0}
$$

for all $x \in \mathbf{E}$. By the same argument as above $\psi$ is also twice Fréchet differentiable and (2.5) follows.

By identifying $H_{\mu}$ with its dual $H_{\mu}^{*}$, the map $D_{H_{\mu}}^{2} \psi(x)$ defines a bounded linear operator on $H_{\mu}$. The following lemma shows that it is actually a Hilbert-Schmidt operator. The proof follows the same argument as in the Hilbert-space case, see e.g. Da Prato and Zabczyk (2002, Chapter 3). We include the proof for the sake of completeness.

Lemma 2.7 For each $x \in \mathbf{E}$ we have $D_{H_{\mu}}^{2} \psi(x) \in \mathcal{T}_{2}\left(H_{\mu}\right)$ and

$$
\left\|D_{H_{\mu}}^{2} \psi(x)\right\|_{\mathcal{T}_{2}\left(H_{\mu}\right)} \leq \sqrt{2}|\varphi|_{0} .
$$

If $\varphi \in C_{b}^{1}(\mathbf{E})$ we have

$$
\left\|D_{H_{\mu}}^{2} \psi(x)\right\|_{\mathcal{T}_{2}\left(H_{\mu}\right)} \leq|\varphi|_{1}
$$

Proof. Let $\left(e_{i}\right)_{i}$ be an orthonormal basis of $H_{\mu}$ and let $x \in \mathbf{E}$ be fixed. Let us prove first the case $\varphi \in C_{b}^{1}(\mathbf{E})$. By the same argument used in the proof of (2.4) one can derive

$$
\left[D_{H_{\mu}}^{2} \psi(x) y_{1}, y_{2}\right]=\int_{\mathbf{E}}\left[D \varphi(x+z), y_{1}\right]_{H_{\mu}} \phi_{\mu}\left(y_{2}\right)(z) \mu(d z), \quad y_{1}, y_{2} \in H_{\mu} .
$$

Since the map $\phi_{\mu}$ is an isometry from $H_{\mu}$ to $L^{2}(\mathbf{E}, \mu)$, the random variables $\phi_{\mu}\left(e_{k}\right), k \in \mathbb{N}$, form a complete or- 
thonormal system in $L^{2}(\mathbf{E}, \mu)$ and by Parseval identity and dominated convergence we get

$$
\begin{aligned}
\left\|D_{H_{\mu}}^{2} \psi(x)\right\|_{\mathcal{T}_{2}\left(H_{\mu}\right)}^{2} & =\sum_{i=1}^{\infty}\left|D_{H_{\mu}}^{2} \psi(x) e_{i}\right|_{H_{\mu}}^{2}=\sum_{i, k=1}^{\infty}\left|\left[D_{H_{\mu}}^{2} \psi(x) e_{i}, e_{k}\right]_{H_{\mu}}\right|^{2} \\
& =\sum_{i, k=1}^{\infty}\left|\left\langle\left[D \varphi(x+\cdot), e_{i}\right]_{H_{\mu}}, \phi_{\mu}\left(e_{k}\right)\right\rangle_{L^{2}(\mathbf{E}, \mu)}\right|^{2} \\
& =\sum_{i=1}^{\infty}\left\|\left[D \varphi(x+\cdot), e_{i}\right]_{H_{\mu}}\right\|_{L^{2}(\mathbf{E}, \mu)}^{2} \\
& =\int_{\mathbf{E}} \sum_{i=1}^{\infty}\left|\left[D \varphi(x+z), e_{i}\right]_{H_{\mu}}\right|^{2} \mu(d z) \\
& =\int_{\mathbf{E}}|D \varphi(x+z)|_{H_{\mu}}^{2} \mu(d z) \\
& \leq|\varphi|_{1}^{2}
\end{aligned}
$$

and (2.11) follows. For the general case $\varphi \in \mathcal{B}_{b}(\mathbf{E})$, we define the random variables

$$
\zeta_{i, k}:= \begin{cases}\frac{1}{\sqrt{2}}\left(\phi_{\mu}\left(e_{i}\right)^{2}-1\right), & \text { if } i=k, \\ \phi_{\mu}\left(e_{i}\right) \phi_{\mu}\left(e_{k}\right), & \text { if } i \neq k .\end{cases}
$$

Since $\phi_{\mu}\left(e_{k}\right), k \in \mathbb{N}$, are independent Gaussian random variables with mean 0 and variance 1 , we get

$$
\left\langle\zeta_{i, k}, \zeta_{i^{\prime}, k^{\prime}}\right\rangle_{L^{2}(\mathbf{E}, \mu)}=0, \quad \text { for }(i, k) \neq\left(i^{\prime}, k^{\prime}\right)
$$

and

$$
\begin{aligned}
\left\|\zeta_{i, k}\right\|_{L^{2}(\mathbf{E}, \mu)}^{2} & =\mathbb{E} \zeta_{i, k}^{2}=\mathbb{E}\left(\phi_{\mu}\left(e_{i}\right)^{2} \phi_{\mu}\left(e_{k}\right)^{2}\right)=1, \quad i \neq k \\
\left\|\zeta_{i, i}\right\|_{L^{2}(\mathbf{E}, \mu)}^{2} & =\mathbb{E} \zeta_{i, i}^{2}=\frac{1}{2} \mathbb{E}\left(\phi_{\mu}\left(e_{i}\right)^{4}-2 \phi_{\mu}\left(e_{i}\right)^{2}+1\right)=\frac{1}{2}(3-2+1)=1,
\end{aligned}
$$

i.e. the system $\left\{\zeta_{i, k}: i, k \in \mathbb{N}\right\}$ is orthonormal in $L^{2}(\mathbf{E}, \mu)$. Recalling (2.5), for $i, k \in \mathbb{N}$ we have

$$
\left[D_{H_{\mu}}^{2} \psi(x) e_{i}, e_{k}\right]_{H_{\mu}}= \begin{cases}\sqrt{2}\left\langle\beta, \zeta_{i, i}\right\rangle_{L^{2}(\mathbf{E}, \mu)}, & \text { if } i=k, \\ \left\langle\beta, \zeta_{i, k}\right\rangle_{L^{2}(\mathbf{E}, \mu)}, & \text { if } i \neq k,\end{cases}
$$

where $\beta(z):=\varphi(x+z)$. Thus, from the Parseval identity and Bessel inequality it follows that

$$
\begin{aligned}
\left\|D_{H_{\mu}}^{2} \psi(x)\right\|_{\mathcal{T}_{2}\left(H_{\mu}\right)}^{2} & =\sum_{i=1}^{\infty}\left|D_{H_{\mu}}^{2} \psi(x) e_{i}\right|_{H_{\mu}}^{2}=\sum_{i, k=1}^{\infty}\left|\left[D_{H_{\mu}}^{2} \psi(x) e_{i}, e_{k}\right]_{H_{\mu}}\right|^{2} \\
& =2 \sum_{i=1}^{\infty}\left|\left\langle\beta, \zeta_{i, i}\right\rangle_{L^{2}(\mathbf{E}, \mu)}\right|^{2}+\sum_{\substack{i, k=1 \\
i \neq k}}^{\infty}\left|\left\langle\beta, \zeta_{i, k}\right\rangle_{L^{2}(\mathbf{E}, \mu)}\right|^{2} \\
& \leq 2 \sum_{i, k=1}^{\infty}\left|\left\langle\beta, \zeta_{i, k}\right\rangle_{L^{2}(\mathbf{E}, \mu)}\right|^{2} \\
& \leq 2\|\beta\|_{L^{2}(\mathbf{E}, \mu)}^{2} \\
& \leq 2|\varphi|_{0}^{2} .
\end{aligned}
$$

\section{Stochastic Integration of Deterministic Operator-Valued Functions in Banach Spaces}

In this section we review some of the results from van Neerven and Weis (2005a) on stochastic integration of deterministic operator valued functions with respect to a cylindrical Wiener process. From this point onwards 
$(\Omega, \mathcal{F}, \mathbb{P})$ is a fixed probability space endowed with a filtration $\mathbb{F}=\left\{\mathcal{F}_{t}\right\}_{t \geq 0},\left(\gamma_{n}\right)_{n}$ is a sequence of real-valued standard Gaussian random variables and $\left(\mathbf{H},[\cdot, \cdot]_{\mathbf{H}}\right)$ is a separable Hilbert space.

Definition 3.1 Let $W(\cdot)=\{W(t)\}_{t \geq 0}$ be a family of bounded linear operators from $\mathbf{H}$ into $L^{2}(\Omega ; \mathbb{R})$. W(.) is called a $\mathbf{H}$-cylindrical Wiener process (with respect to the filtration $\mathbb{F}$ ) iff

(i) $\mathbb{E}\left[W(t) h_{1} W(t) h_{2}\right]=t\left[h_{1}, h_{2}\right]_{\mathbf{H}}$, for all $h_{1}, h_{2} \in \mathbf{H}$ and $t \geq 0$

(ii) For each $h \in \mathbf{H}$, the process $\{W(t) h\}_{t \geq 0}$ is a standard real-valued $\mathbb{F}$-Brownian motion.

Definition 3.2 A linear bounded operator $\Phi: \mathbf{H} \rightarrow \mathbf{E}$ is said to be $\gamma$-radonifying iff there exists an orthonormal basis $\left(e_{n}\right)_{n \geq 1}$ of the Hilbert space $\mathbf{H}$ such that the random sum $\sum_{n \geq 1} \gamma_{n} \Phi e_{n}$ converges in $L^{2}(\Omega ; \mathbf{E})$.

We denote by $\gamma(\mathbf{H}, \mathbf{E})$ the class of $\gamma$-radonifying operators from $\mathbf{H}$ into $\mathbf{E}$. It can be proved that this a Banach space with the norm

$$
\|\Phi\|_{\gamma(\mathbf{H}, \mathbf{E})}^{2}:=\mathbb{E}\left|\sum_{n \geq 1} \gamma_{n} \Phi e_{n}\right|_{\mathbf{E}}^{2}, \quad \Phi \in \gamma(\mathbf{H}, \mathbf{E}) .
$$

This definition is independent of the choice of the orthonormal basis $\left(e_{n}\right)_{n \geq 1}$ of $\mathbf{H}$. Moreover, $\gamma(\mathbf{H}, \mathbf{E})$ is embedded continuously into $\mathcal{L}(\mathbf{H}, \mathbf{E})$ and is an operator ideal in the sense that if $\mathbf{H}^{\prime}$ and $\mathbf{E}^{\prime}$ are Hilbert and Banach spaces respectively such that $S_{1} \in \mathcal{L}\left(\mathbf{H}^{\prime}, \mathbf{H}\right)$ and $S_{2} \in \mathcal{L}\left(\mathbf{E}, \mathbf{E}^{\prime}\right)$ then $\Phi \in \gamma(\mathbf{H}, \mathbf{E})$ implies $S_{2} \Phi S_{1} \in \gamma\left(\mathbf{H}^{\prime}, \mathbf{E}^{\prime}\right)$ with

$$
\left\|S_{2} \Phi S_{1}\right\|_{\gamma\left(\mathbf{H}^{\prime}, \mathbf{E}^{\prime}\right)} \leq\left\|S_{2}\right\|_{\mathcal{L}\left(\mathbf{E}, \mathbf{E}^{\prime}\right)}\|\Phi\|_{\gamma(\mathbf{H}, \mathbf{E})}\left\|S_{1}\right\|_{\mathcal{L}\left(\mathbf{H}^{\prime}, \mathbf{H}\right)}
$$

It can also be proved that $\Phi$ is $\gamma$-radonifying if and only if $\Phi \Phi^{*}$ is the covariance operator of a centered Gaussian measure on $\mathcal{B}(\mathbf{E})$, and if $\mathbf{E}$ is a Hilbert space, then $\Phi$ is $\gamma$-radonifying iif and only if $\Phi$ is a Hilbert-Schmidt operator from $\mathbf{H}$ into $\mathbf{E}$ (see e.g. van Neerven (2008) and the references therein).

The $\gamma$-radonifying property in the following example goes back to Brzeźniak (1996), and will be used later in our main Example 5.3. For the sake of completeness, we include a proof which follows closely arguments from (van Neerven 2008, Chapter 15).

Example 3.3 For $p \geq 1$, let $\Delta_{p}$ denote the realization of $-\frac{d^{2}}{d \xi^{2}}$ in $L^{p}(0,1)$ with zero-Dirichlet boundary conditions. Then, for $\sigma \in\left(\frac{1}{4}, 1\right)$, the identity operator on $D\left(\Delta_{2}\right)$ extends to a continuous embedding $j: D\left(\Delta_{2}\right) \hookrightarrow D\left(\Delta_{p}^{1-\sigma}\right)$ that is $\gamma$-radonifying.

Proof. The functions $e_{n}(\xi)=\sqrt{2} \sin (n \pi \xi), n \geq 1$, form an orthonormal basis of eigenfunctions for $\Delta_{2}$ with eigenvalues $\lambda_{n}=(n \pi)^{2}$. If we endow $D\left(\Delta_{2}\right)$ with the equivalent Hilbert norm $|y|_{D\left(\Delta_{2}\right)}:=\left|\Delta_{2} y\right|_{L^{2}(0,1)}$, the functions $\lambda_{n}^{-1} e_{n}$ form an orthonormal basis for $D\left(\Delta_{2}\right)$.

Let $\left(\gamma_{n}\right)_{n}$ be a Gaussian sequence on a probability space $(\Omega, \mathcal{F}, \mathbb{P})$. Then, we have

$$
\mathbb{E}\left|\sum_{n \geq 1} \gamma_{n} \lambda_{n}^{-1} e_{n}\right|_{D\left(\Delta_{p}^{1-\sigma}\right)}^{2}=\mathbb{E}\left|\sum_{n \geq 1} \gamma_{n} \lambda_{n}^{-1} \Delta_{p}^{1-\sigma} e_{n}\right|_{L^{p}(0,1)}^{2}=\mathbb{E}\left|\sum_{n \geq 1} \gamma_{n}(n \pi)^{-2 \sigma} e_{n}\right|_{L^{p}(0,1)}^{2}
$$

Using Hölder's inequality, we have

$$
\begin{aligned}
\mathbb{E}\left|\sum_{n=N}^{M} \gamma_{n}(n \pi)^{-2 \sigma} e_{n}\right|_{L^{p}(0,1)}^{2} & \leq\left(\mathbb{E}\left|\sum_{n=N}^{M} \gamma_{n}(n \pi)^{-2 \sigma} e_{n}\right|_{L^{p}(0,1)}^{p}\right)^{2 / p} \\
& =\left(\mathbb{E} \int_{0}^{1}\left|\sum_{n=N}^{M} \gamma_{n}(n \pi)^{-2 \sigma} e_{n}(\xi)\right|^{p} d \xi\right)^{2 / p} \\
& =\left(\int_{0}^{1} \mathbb{E}\left|\sum_{n=N}^{M} \gamma_{n}(n \pi)^{-2 \sigma} e_{n}(\xi)\right|^{p} d \xi\right)^{2 / p} \\
& \leq\left(\int_{0}^{1}\left(\mathbb{E}\left|\sum_{n=N}^{M} \gamma_{n}(n \pi)^{-2 \sigma} e_{n}(\xi)\right|^{2}\right)^{p / 2} d \xi\right)^{2 / p}
\end{aligned}
$$

By Kahane-Khintchine inequality, there exists a constant $c^{\prime}$ such that

$$
\mathbb{E}\left|\sum_{n=N}^{M} \gamma_{n}(n \pi)^{-2 \sigma} e_{n}(\xi)\right|^{2} \leq c^{\prime} \sum_{n=N}^{M}\left|(n \pi)^{-2 \sigma} e_{n}(\xi)\right|^{2}=c^{\prime} \sum_{n=N}^{M}(n \pi)^{-4 \sigma} e_{n}^{2}(\xi)
$$


Hence, we obtain

$$
\mathbb{E}\left|\sum_{n=N}^{M} \gamma_{n}(n \pi)^{-2 \sigma} e_{n}\right|_{L^{p}(0,1)}^{2} \leq\left(\int_{0}^{1}\left[c^{\prime} \sum_{n=N}^{M}(n \pi)^{-4 \sigma} e_{n}^{2}(\xi)\right]^{p / 2} d \xi\right)^{2 / p}=c^{\prime}\left|\sum_{n=N}^{M}(n \pi)^{-4 \sigma} e_{n}^{2}\right|_{L^{p / 2}(0,1)} \leq c^{\prime} \sum_{n=N}^{M}(n \pi)^{-4 \sigma}\left|e_{n}^{2}\right|_{L^{p / 2}(0,1)}
$$

Since $\left|e_{n}^{2}\right|_{L^{p / 2}(0,1)}=\left|e_{n}\right|_{L^{p}(0,1)}^{2} \leq 2$ for all $n \geq 1$, it follows that

$$
\mathbb{E}\left|\sum_{n=N}^{M} \gamma_{n}(n \pi)^{-2 \sigma} e_{n}\right|_{L^{p}(0,1)}^{2} \leq 2 c_{p}^{\prime} \sum_{n=N}^{M}(n \pi)^{-4 \sigma}
$$

The right-hand side of the last inequality tends to 0 as $N, M \rightarrow \infty$ since $\sigma>\frac{1}{4}$. Therefore, the right-hand side of (3.1) is finite, and the claim follows.

Before we discuss the integral for $\mathcal{L}(\mathbf{H}, \mathbf{E})$-valued functions, we observe that we can integrate certain $\mathbf{H}$-valued functions with respect to a $\mathbf{H}$-cylindrical Wiener process $W(\cdot)$. For a step function of the form $\psi=\mathbf{1}_{(s, t]} y$ with $y \in \mathbf{H}$ we define

$$
\int_{0}^{T} \psi(r) d W(r):=W(t) y-W(s) y .
$$

This extends to arbitrary step functions $\psi$ by linearity, and a standard computation shows that

$$
\mathbb{E}\left|\int_{0}^{T} \psi(r) d W(r)\right|_{\mathbb{R}}^{2}=\int_{0}^{T}|\psi(t)|_{\mathbf{H}}^{2} d t
$$

Since the set of step functions $L_{\text {step }}^{2}(0, T ; \mathbf{H})$ is dense in $L^{2}(0, T ; \mathbf{H})$, the map

$$
I_{T}: L_{\text {step }}^{2}(0, T ; \mathbf{H}) \ni \psi \mapsto \int_{0}^{T} \psi(t) d W(t) \in L^{2}(\Omega ; \mathbb{R})
$$

extends to a (linear!) isometry from $L^{2}(0, T ; \mathbf{H})$ into $L^{2}(\Omega)$. We now define the stochastic integral for deterministic $\mathcal{L}(\mathbf{H}, \mathbf{E})$-valued functions with respect to $W(\cdot)$.

\section{Definition 3.4}

1) A function $\Phi:(0, T) \rightarrow \mathcal{L}(\mathbf{H}, \mathbf{E})$ is said to belong scalarly to $L^{2}(0, T ; \mathbf{H})$ if the map

$$
[0, T] \ni t \mapsto \Phi(t)^{*} x^{*} \in \mathbf{H}
$$

belongs to $L^{2}(0, T ; \mathbf{H})$ for every $x^{*} \in \mathbf{E}^{*}$.

2) A function $\Phi:(0, T) \rightarrow \mathcal{L}(\mathbf{H}, \mathbf{E})$ is said to be stochastically integrable with respect to $W(\cdot)$ if it belongs scalarly to $L^{2}(0, T ; \mathbf{H})$ and for all $A \subset(0, T)$ measurable there exists a random variable $Y_{A} \in L^{2}(\Omega, \mathcal{F}, \mathbb{P} ; \mathbf{E})$ such that

$$
\left\langle Y_{A}, x^{*}\right\rangle=\int_{0}^{T} \mathbf{1}_{A}(t) \Phi(t)^{*} x^{*} d W(t), \quad \mathbb{P}-\text { a.s., for all } x^{*} \in \mathbf{E}^{*} .
$$

We denote

$$
\int_{A} \Phi(t) d W(t):=Y_{A}
$$

By Fernique's theorem, the $\mathbf{E}$-valued random variables $Y_{A}$ are uniquely determined almost everywhere and Gaussian. In particular $Y_{A} \in L^{p}(\Omega ; \mathbf{E})$ for all $p \geq 1$.

For a function $\Phi:(0, T) \rightarrow \mathcal{L}(\mathbf{H}, \mathbf{E})$ that belongs scalarly to $L^{2}(0, T ; \mathbf{H})$ we define an operator $R_{\Phi}: L^{2}(0, T ; \mathbf{H}) \rightarrow$ $\mathbf{E}^{* *}$ by

$$
\left\langle x^{*}, R_{\Phi} f\right\rangle:=\int_{0}^{T}\left[\Phi(t)^{*} x^{*}, f(t)\right]_{\mathbf{H}} d t, \quad f \in L^{2}(0, T ; \mathbf{H}), \quad x^{*} \in \mathbf{E}^{*} .
$$

Observe that $I_{\Phi}$ is the adjoint of the operator

$$
\mathbf{E}^{*} \ni x^{*} \mapsto \Phi(t)^{*} x^{*} \in L^{2}(0, T ; \mathbf{H}) .
$$


If $\Phi(\cdot) y$ is strongly measurable for all $y \in \mathbf{H}$ then $R_{\Phi}$ maps $L^{2}(0, T ; \mathbf{H})$ into $\mathbf{E}$. The following theorem characterizes the class of stochastically integrable functions with respect to the $\mathbf{H}$-cylindrical Wiener process $W(\cdot)$.

Theorem 3.5 (van Neerven \& Weis, 2005a, Theorem 4.2) Let $\mathbf{E}$ be a separable Banach space. For a function $\Phi$ : $(0, T) \rightarrow \mathcal{L}(\mathbf{H}, \mathbf{E})$ that belongs scalarly to $L^{2}(0, T ; \mathbf{H})$ the following assertions are equivalent

1) $\Phi$ is stochastically integrable with respect to $W(\cdot)$;

2) There exists an $\mathbf{E}$-valued random variable $Y$ such that for all $x \in \mathbf{E}^{*}$

$$
\left\langle Y, x^{*}\right\rangle=\int_{0}^{T} \Phi(t)^{*} x^{*} d W(t), \quad \mathbb{P}-\text { a.s., } \forall x^{*} \in \mathbf{E}^{*} .
$$

3) There exists a centered Gaussian measure $\mu$ on $\mathbf{E}$ with covariance operator $\mathbf{C} \in \mathcal{L}\left(\mathbf{E}, \mathbf{E}^{*}\right)$ such that for all $x \in \mathbf{E}^{*}$

$$
\left\langle\mathbf{C} x^{*}, x^{*}\right\rangle=\int_{0}^{T}\left|\Phi(t)^{*} x^{*}\right|_{\mathbf{H}}^{2} d t
$$

4) There exist a separable Hilbert space $\mathfrak{H}$ a linear bounded operator $S \in \gamma(\mathfrak{H}, \mathbf{E})$ such that for all $x \in \mathbf{E}^{*}$

$$
\int_{0}^{T}\left|\Phi(t)^{*} x^{*}\right|_{\mathbf{H}}^{2} d t \leq\left|S^{*} x^{*}\right|_{\mathfrak{H}}^{2} .
$$

5) $R_{\Phi}$ maps $L^{2}(0, T ; \mathbf{H})$ into $\mathbf{E}$ and $R_{\Phi} \in \gamma\left(L^{2}(0, T ; \mathbf{H}) ; \mathbf{E}\right)$.

Moreover, for all $y \in \mathbf{H}$ the function $\Phi(\cdot) y$ is stochastic integrable with respect to $W(\cdot) y$ and we have the series representation

$$
\int_{0}^{T} \Phi(t) d W(t)=\sum_{n=1}^{\infty} \int_{0}^{T} \Phi(t) e_{n} d W(t) e_{n}
$$

where $\left(e_{n}\right)_{n \geq 1}$ is any orthonormal basis for $\mathbf{H}$. The series converges $\mathbb{P}-$ a.s and in $L^{p}(\Omega ; \mathbf{E})$ for all $p \in[0, \infty)$. The measure $\mu$ is the distribution of $\int_{0}^{T} \Phi(t) d W(t)$ and we have the isometry

$$
\mathbb{E}\left|\int_{0}^{T} \Phi(t) d W(t)\right|_{\mathbf{E}}=\left\|R_{\Phi}\right\|_{\gamma\left(L^{2}(0, T ; \mathbf{H}) ; \mathbf{E}\right)}
$$

We conclude this section with a sufficient condition for stochastic integrability in spaces of type 2 (see e.g. van Neerven \& Weis, 2005a, Theorem 4.7 or 2005b, Theorem 5.1).

Definition $3.6 \mathrm{E}$ is said to be of type 2 iff there exists $K_{2}>0$ such that

$$
\mathbb{E}\left|\sum_{i=1}^{n} \epsilon_{i} x_{i}\right|_{\mathbf{E}}^{2} \leq K_{2} \sum_{i=1}^{n}\left|x_{i}\right|_{\mathbf{E}}^{2}
$$

for any finite sequence $\left\{x_{i}\right\}_{i=1}^{n}$ of elements of $\mathbf{E}$ and for any finite sequence $\left\{\epsilon_{i}\right\}_{i=1}^{n}$ of $\{-1,1\}$-valued symmetric i.i.d. random variables.

Theorem 3.7 Let $\mathbf{E}$ be a separable real Banach space of type 2. If $\Phi:(0, T) \rightarrow \mathcal{L}(\mathbf{H}, \mathbf{E})$ belongs scalarly to $L^{2}(0, T ; \mathbf{H})$, for almost all $t \in(0, T)$ we have $\Phi(t) \in \gamma(\mathbf{H}, \mathbf{E})$, and

$$
\int_{0}^{T}\|\Phi(t)\|_{\gamma(\mathbf{H}, \mathbf{E})}^{2} d t<\infty
$$

then $\Phi$ is stochastically integrable with respect to $W(\cdot)$ and

$$
\mathbb{E}\left|\int_{0}^{T} \Phi(t) d W(t)\right|_{\mathbf{E}}^{2} \leq K_{2}^{2} \int_{0}^{T}\|\Phi(t)\|_{\gamma(\mathbf{H}, \mathbf{E})}^{2} d t .
$$

Proof. See Theorem 5.1 in van Neerven and Weis (2005b). 


\section{Parabolic Evolution Families}

Since there is no unified theory for parabolic evolution families and non-autonomous evolution equations, we restrict in this paper to the class of parabolic problems and setting introduced by Acquistapace and Terreni (1987). We start this section by recalling the definition of positive operators with bounded imaginary powers.

Definition 4.1 Let $A$ be a densely defined closed linear operator on a Banach space $\mathbf{E}$. The operator $A$ is said to be positive if $(-\infty, 0] \subset \rho(A)$ and if there exists a constant $k \geq 1$ with

$$
\left\|(w I+A)^{-1}\right\|_{\mathcal{L}(\mathbf{E})} \leq \frac{k}{1+w}, \quad \text { for all } w \geq 0 .
$$

It is well known that every positive operator $A$ admits (not necessarily bounded) fractional powers $A^{z}$ of any order $z \in \mathbb{C}$, see e.g. Amann (1995, Chapter III, Section 4.6).

Definition 4.2 We define the class of operators with bounded imaginary powers on $\mathbf{E}$ with parameter $\phi \in[0, \pi)$, denoted $\operatorname{BIP}(\phi, \mathbf{E})$, as the class of positive operators $A$ on $\mathbf{E}$ such that $A^{i r} \in \mathcal{L}(\mathbf{E})$ for all $r \in \mathbb{R}$ and there exists a constant $k>0$ with

$$
\left\|A^{i r}\right\|_{\mathcal{L}(\mathbf{E})} \leq k e^{\phi|r|}, r \in \mathbb{R} .
$$

For each $t \in[0, T]$ let $A(t)$ be a densely defined closed linear operator on a Banach space E. For each $s \in[0, T]$, consider the following non-autonomous Cauchy problem

$$
\begin{aligned}
y^{\prime}(t)+A(t) y(t) & =0, \quad t \in(s, T] \\
y(s) & =x \in \mathbf{E} .
\end{aligned}
$$

Definition 4.3 We say that $y \in C((s, T] ; \mathbf{E}) \cap C^{1}((s, T] ; \mathbf{E})$ is a classical solution of $(4.1)$ if $y(t) \in D(A(t))$ for all $t \in(s, T]$ and (4.1) holds.

Definition 4.4 We say that a classical solution $y$ of (4.1) is also a strict solution if in addition $y \in C^{1}([s, T]$; $\mathbf{E})$, $x \in D(A(s))$ and $A(t) y(t) \rightarrow A(s) x$ as $t \rightarrow s$.

We say that condition (AT) is satisfied if the two following conditions hold

(AT1) There exist constants $w \in \mathbb{R}, K \geq 0$ and $\phi \in\left(\frac{\pi}{2}, \pi\right)$ such that

$$
\Sigma(\phi, w):=\{w\} \cup\{\lambda \in \mathbb{C} \backslash\{w\}:|\arg (\lambda-w)| \leq \phi\} \subset \rho(-A(t))
$$

and for all $\lambda \in \Sigma(\phi, w)$ and $t \in[0, T]$,

$$
\left\|(A(t)+\lambda I)^{-1}\right\|_{\mathcal{L}(\mathbf{E})} \leq \frac{K}{1+|\lambda-w|} .
$$

(AT2) There exist constants $L \geq 0$ and $\mu, v \in(0,1)$ with $\mu+v>1$ such that for all $\lambda \in \Sigma(\phi, 0)$ and $s, t \in[0, T]$,

$$
\left\|A_{w}(t)\left(A_{w}(t)+\lambda I\right)^{-1}\left[A_{w}(t)^{-1}-A_{w}(s)^{-1}\right]\right\|_{\mathcal{L}(\mathbf{E})} \leq L \frac{|t-s|^{\mu}}{(|\lambda|+1)^{v}},
$$

where $A_{w}(t):=A(t)+w I$.

Operators satisfying (AT1) are called sectorial (of type $(\phi, K, w)$ ). Equation (4.1) is called parabolic because of the sectoriality of the operators $A(t)$.

If Assumption (AT1) is satisfied and the domains are constant i.e. $D(A(t))=D(A(0))$ for all $t \in[0, T]$, and the map $[0, T] \ni t \mapsto A(t) \in \mathcal{L}(D(A(0)), \mathbf{E})$ is Hölder continuous with exponent $\eta$, then (AT2) is satisfied with $\mu=\eta$ and $v=1$, see e.g. (Acquistapace \& Terreni, 1987, Section 7). In this case such conditions reduce to the theory of Sobolevskii and Tanabe for constant domains (see e.g. Pazy, 1983 or Tanabe, 1979).

In what follows we denote $\mathfrak{T}:=\left\{(t, s) \in[0, T]^{2}: s \leq t\right\}$.

Definition 4.5 A family of bounded operators $\{S(t, s)\}_{(t, s) \in \mathfrak{T}}$ on $\mathbf{E}$ is called a strongly continuous evolution family if the following hold

(1) $S(t, t)=I$, for all $t \in[0, T]$. 
(2) $S(t, s)=S(t, r) S(r, s)$ for all $0 \leq s \leq r \leq t \leq T$.

(3) The mapping $\mathfrak{T} \ni(t, s) \mapsto S(t, s) \in \mathcal{L}(\mathbf{E})$ is strongly continuous.

We say that the family $\{S(t, s)\}_{(t, s) \in \mathcal{T}}$ solves non-autonomous Cauchy problem (4.1) if there exist a family $\left(Y_{s}\right)_{s \in[0, T]}$ of dense subspaces of $\mathbf{E}$ such that for all $(s, t) \in \mathfrak{T}$ we have

$$
S(t, s) Y_{s} \subset Y_{t} \subset D(A(t))
$$

and the map $y(t)=S(t, s) x$ is a strict solution of (4.1) for every $x \in Y_{s}$. In this case we say that $\{-A(t), D(A(t))\}_{t \in[0, T]}$ (or simply $\left.\{-A(t)\}_{t \in[0, T]}\right)$ generates the evolution family $\{S(t, s)\}_{(t, s) \in \mathfrak{T}}$.

Under the condition (AT) we have the following well-known result, see e.g. Acquistapace and Terreni (1987, Theorems 6.1-6.4) and Yagi (1991, Theorem 2.1).

Theorem 4.6 If condition (AT) holds then there exists a unique strongly continuous evolution family $\{S(t, s)\}_{(t, s) \in \mathfrak{T}}$ that solves the non-autonomous Cauchy problem (4.1) with $Y_{t}=D(A(t))$ and for all $x \in \mathbf{E}$, the map $y(t)=S(t, s) x$ is a classical solution of (4.1). Moreover, $\{S(t, s)\}_{(t, s) \in \mathfrak{T}}$ is continuous on $0 \leq s<t \leq T$ and there exists a constant $C>0$ such that for all $0 \leq s<t \leq T$ and $\theta \in[0,1]$,

$$
\begin{aligned}
& \left\|(A(t)+w I)^{\theta} S(t, s)\right\|_{\mathcal{L}(\mathbf{E})} \leq C(t-s)^{-\theta} \\
& \left\|S(t, s)-e^{-(t-s) A(s)}\right\|_{\mathcal{L}(\mathbf{E})} \leq C(t-s)^{\mu+\nu-1}
\end{aligned}
$$

Moreover, for all $\theta \in(0, \mu)$ and $x \in D\left((A(t)+w I)^{\theta}\right)$ we have

$$
\left|S(t, s)(A(t)+w I)^{\theta} x\right|_{\mathbf{E}} \leq C(\mu-\theta)^{-1}(t-s)^{-\theta}|x|_{\mathbf{E}} .
$$

\section{Backward Ornstein-Uhlenbeck Transition Evolution Operators}

Let $\{-A(t)\}_{t \in[0, T]}$ be the generator of an evolution family $\{S(t, s)\}_{(t, s) \in \mathfrak{T}}$ on $\mathbf{E}$ and let $\{G(t)\}_{t \in[0, T]}$ be closed operators from a constant domain $D(G) \subset \mathbf{H}$ into $\mathbf{E}$. We start this section by discussing the existence of mild solutions to the non-autonomous linear stochastic equation

$$
\begin{aligned}
d Z(t)+A(t) Z(t) d t & =G(t) d W(t), \quad t \in[s, T], \\
Z(s) & =x_{0} \in \mathbf{E},
\end{aligned}
$$

with moving time origin $s \in[0, T]$ and initial data $x_{0} \in \mathbf{E}$.

Definition 5.1 We say that an $\mathbf{E}$-valued process $Z(\cdot)$ is a mild solution of (5.1) if for all $(t, s) \in \mathfrak{T}$ the mapping $S(t, s) G(s)$ has a continuous extension to a bounded operator from $\mathbf{H}$ into $\mathbf{E}$, which we will also denote by $S(t, s) G(s)$, such that the operator-valued function $(s, t) \ni r \mapsto S(t, r) G(r) \in \mathcal{L}(\mathbf{H}, \mathbf{E})$ is stochastically integrable on the interval $(s, t)$ and

$$
Z(t)=S(t, s) x_{0}+\int_{s}^{t} S(t, r) G(r) d W(r), \quad \mathbb{P}-\text { a.s }
$$

We know from Theorem 3.5 that existence of a mild solution for (5.1) follows from the following condition

Assumption A.1 For each $(t, s) \in \mathfrak{T}$ the mapping $S(t, s) G(s): D(G) \rightarrow \mathbf{E}$ extends to a bounded linear operator from $\mathbf{H}$ into $\mathbf{E}$, also denoted by $S(t, s) G(s)$, such that the positive symmetric operator $\mathbf{C}_{t, s} \in \mathcal{L}\left(\mathbf{E}^{*}, \mathbf{E}\right)$ defined by

$$
\left\langle\mathbf{C}_{t, s} x^{*}, y^{*}\right\rangle:=\int_{s}^{t}\left\langle S(t, r) G(r)(S(t, r) G(r))^{*} x^{*}, y^{*}\right\rangle d r, \quad x^{*}, y^{*} \in \mathbf{E}^{*}
$$

is the covariance operator of a centered Gaussian measure $\mu_{t, s}$ on $\mathbf{E}$.

Notation For each $(t, s) \in \mathfrak{T}$, let $\left(H_{t, s,}[\cdot, \cdot]_{H_{t, s}}\right)$ denote the Reproducing Kernel Hilbert Space associated with the positive symmetric operator $\mathbf{C}_{t, s}$ defined by (5.2), and let $i_{t, s}$ denote the inclusion mapping from $H_{t, s}$ into $\mathbf{E}$.

Example 5.2 Let $\mathbf{E}$ be a type-2 Banach space and suppose that for each $(t, s) \in \mathfrak{T}$ we have $S(t, s) G(s) \in \gamma(\mathbf{H}, \mathbf{E})$ and

$$
\int_{0}^{T}|S(t, s) G(s)|_{\gamma(\mathbf{H}, \mathbf{E})}^{2} d t<+\infty
$$


Then, by Theorem 3.7, Assumption A.1 holds.

For the next example, consider the following linear parabolic second-order stochastic PDE perturbed by additive space-time white noise on $[0, T] \times(0,1)$,

$$
\begin{aligned}
\frac{\partial X}{\partial t}(t, \xi)+\left(\mathcal{A}_{t} X\right)(t, \xi) & =g(t, \xi) \frac{\partial w}{\partial t}(t, \xi), \\
X(t, 0)=X(t, 1) & =0 \\
X(0, \cdot) & =x_{0}(\cdot)
\end{aligned}
$$

where, for each $t \in[0, T], \mathcal{A}_{t}$ is the second-order differential operator

$$
\left(\mathcal{A}_{t} x\right)(\xi):=-a(t, \xi) \frac{d^{2} x}{d \xi^{2}}(\xi)+b(t, \xi) \frac{d x}{d \xi}(\xi)+c(t, \xi) x(\xi), \quad \xi \in(0,1)
$$

with $a, b, \mathbf{C} \in C^{\mu}([0, T] ; C([0,1]))$ and $a \in C^{\varepsilon}([0,1] ; C([0, T]))$ for $\mu \in\left(\frac{1}{4}, 1\right]$ and $\varepsilon>0$ fixed. Assume further that $\inf _{t \in[0, T], \xi \in[0,1]} a(t, \xi)>0$.

For $p \geq 2$ and $t \in[0, T]$, let $A_{p}(t)$ denote the realization in $L^{p}(0,1)$ of $\mathcal{A}_{t}$ with zero-Dirichlet boundary conditions,

$$
\begin{aligned}
D\left(A_{p}(t)\right) & :=H^{2, p}(0,1) \cap H_{0}^{1, p}(0,1), \\
A_{p}(t) & :=\mathcal{A}_{t} .
\end{aligned}
$$

It is well-known that for $w$ sufficiently large, the operator $A_{p}(\cdot)+w I$ satisfies (AT) with parameters $\mu$ and $v=1$ (see e.g. Acquistapace \& Terreni, 1987 or Tanabe, 1979). We will assume for simplicity and without loss of generality that $w=0$.

Let $\left\{S_{p}(t, s)\right\}_{(t, s) \in \mathfrak{T}}$ denote the family of evolution operators generated by $\left\{-A_{p}(t)\right\}_{t \in[0, T]}$. Let $g \in L^{1}\left(0, T ; L^{\infty}(0,1)\right)$ be fixed and define, for almost every $t \in[0, T]$, the multiplication operators from $L^{2}(0,1)$ into $L^{p}(0,1)$ as follows

$$
\begin{aligned}
D(G(t)) & :=L^{p}(0,1) \subset L^{2}(0,1) \\
G(t) y & :=\{(0,1) \ni \xi \mapsto g(t, \xi) y(\xi) \in \mathbb{R}\} .
\end{aligned}
$$

Notice that $G(t)$ is not a bounded operator unless $p=2$. However, we can prove the following

Example 5.3 For each $(t, s) \in \mathfrak{T}$ the map $S_{p}(t, s) G(s)$ can be extended to bounded operator from $L^{2}(0,1)$ into $L^{p}(0,1)$ that is $\gamma$-radonifying and satisfies (5.3). Since $L^{p}(0,1)$ has type-2 for $p \geq 2$, from Example 5.2 it follows that Assumption A.1 holds for $\left\{S_{p}(t, s) G(s)\right\}_{(t, s) \in \mathfrak{T}}$ with $\mathbf{H}=L^{2}(0,1)$ and $\mathbf{E}=L^{p}(0,1)$ with $p \geq 2$. Equivalently, Equation (5.4) has a mild solution in $L^{p}(0,1)$.

Proof. The argument of the proof follows closely (Veraar \& Zimmerschied, 2008, Section 5). We show first that if $\sigma>\frac{1}{4}$ then $A_{p}(t)^{-\sigma}$ extends to a bounded operator from $L^{2}(0,1)$ into $L^{p}(0,1)$, which we also denote by $A_{p}(t)^{-\sigma}$, such that

$$
A_{p}(t)^{-\sigma} \in \gamma\left(L^{2}(0,1), L^{p}(0,1)\right) .
$$

We know from Example 3.3 that the identity operator on $D\left(\Delta_{2}\right)$ extends to a continuous embedding $j: D\left(\Delta_{2}\right) \hookrightarrow$ $D\left(\Delta_{p}^{1-\sigma}\right)$ which is $\gamma$-radonifying. Moreover, the family of operators $\left\{A_{p}(t) A_{p}(s)^{-1}: s, t, \in[0, T]\right\}$ is uniformly bounded in $\mathcal{L}\left(L^{p}(0,1)\right)$ (see e.g. Tanabe, 1979, Section 5.2). This implies, in particular, that both domains $D\left(A_{p}(t)\right)$ and $D\left(A_{p}(0)\right)$ coincide with equivalent norms, uniformly in $t \in[0, T]$. Since $D\left(A_{p}(0)\right)=D\left(\Delta_{p}\right)$ with equivalent norms, we conclude that $D\left(A_{p}(t)\right)=D\left(\Delta_{p}\right)$ with equivalent norms uniformly in $t \in[0, T]$.

Using the $\varepsilon$-Hölder continuity assumption on the coefficients of $\mathcal{A}_{t}$, it can be proved that the operators $A_{p}(t)$ belong to $\operatorname{BIP}\left(L^{p}(0,1), \phi\right)$ for some $\phi>0$, see e.g. Denk et al. (2004) or Prüss and Sohr (1993). Hence, by Theorem 1.15.3 in Triebel (1978) we have

$$
D\left(A_{p}(t)^{1-\sigma}\right)=\left[L^{p}(0,1), D\left(A_{p}(t)\right)\right]_{1-\sigma}=\left[L^{p}(0,1), D\left(\Delta_{p}\right)\right]_{1-\sigma}=D\left(\Delta_{p}^{1-\sigma}\right)
$$

isomorphically, with equivalence in norm uniformly in $t \in[0, T]$. Therefore, by the ideal property of $\gamma\left(D\left(\Delta_{2}\right)\right.$, $\left.D\left(\Delta_{p}^{1-\sigma}\right)\right)$, we obtain

$$
A_{p}(t)^{-\sigma}=A_{p}(t)^{1-\sigma} j A_{2}(t)^{-1} \in \gamma\left(L^{2}(0,1), L^{p}(0,1)\right)
$$


with $\left\|A_{p}(t)^{-\sigma}\right\|_{\gamma\left(L^{2}(0,1), L^{p}(0,1)\right)}$ uniformly bounded in $t \in[0, T]$.

Now, from (4.2) it follows that, if $\sigma \in(0, \mu)$ then the map $S_{p}(t, s) A_{p}(s)^{\sigma}$ extends to a bounded operator $S_{p, \sigma}(t, s)$ on $L^{p}(0,1)$ with

$$
\left\|S_{p, \sigma}(t, s)\right\|_{\mathcal{L}\left(L^{p}(0,1)\right)} \leq C(\mu-\sigma)^{-1}(t-s)^{-\sigma} .
$$

Hence, again by the ideal property of $\gamma$-radonifying operators, we conclude that if $\sigma \in\left(\frac{1}{4}, \mu\right)$, for each $(t, s) \in \mathfrak{T}$ the linear mappings

$$
S_{p}(t, s) G(s)=S_{p}(t, s) A_{p}(s)^{\sigma} A_{p}(s)^{-\sigma} G(s)
$$

have a continuous extension to bounded operators from $L^{2}(0,1)$ into $L^{p}(0,1)$ that are $\gamma$-radonifying and satisfy (5.3).

We now introduce the transition evolution operators associated with the linearized Equation (5.1). Suppose that Assumptions (AT) and A.1 are satisfied. Let $\mathcal{B}_{b}(\mathbf{E})$ denote the set of Borel-measurable bounded real-valued functions on $\mathbf{E}$.

Definition 5.4 The Ornstein-Uhlenbeck (OU) transition evolution operators $\{P(s, t)\}_{(t, s) \in \mathfrak{T}}$ associated to Equation (5.1) are defined by

$$
[P(s, t) \varphi](x):=\int_{\mathbf{E}} \varphi(S(t, s) x+z) \mu_{t, s}(d z), \quad x \in \mathbf{E}, \varphi \in \mathcal{B}_{b}(\mathbf{E}), \quad(t, s) \in \mathfrak{T}
$$

Before we discuss the smoothing property of the OU transition operators, we extend to the non-autonomous framework some results by (van Neerven 1998, Section 1) on the relation between the spaces $H_{t, s}$ for different values of $s<t$. The first observation is the following algebraic relation between the operators $\mathbf{C}_{t, s}$, which is immediate from their definition

$$
\mathbf{C}_{t, s}=\mathbf{C}_{t, r}+S(t, r) \mathbf{C}_{r, s} S(t, r)^{*}, \quad 0 \leq s<r<t .
$$

The following is a direct consequence of Proposition 2.3,

Proposition $5.5 H_{t, r} \subset H_{t, s}$ for all $0 \leq s<r<t$.

This combined with the identity $S(t, r) \mathbf{C}_{r, s} S(t, r)^{*}=\mathbf{C}_{t, s}-\mathbf{C}_{t, r}$, implies that $S(t, r)$ maps the linear subspace Range $\mathbf{C}_{r, s} S(t, r)^{*}$ of $H_{r, s}$ into $H_{t, s}$. The next result shows that we actually have $S(t, r) H_{r, s} \subset H_{t, s}$.

Theorem 5.6 For all $0 \leq s<r<t$ we have $S(t, r) H_{r, s} \subset H_{t, s}$. Moreover $\|S(t, r)\|_{\mathcal{L}\left(H_{r, s}, H_{t, s}\right)} \leq 1$.

Proof. For all $x^{*} \in \mathbf{E}^{*}$ we have

$$
\left|\mathbf{C}_{r, s} S(t, r)^{*} x^{*}\right|_{H_{r, s}}^{2}=\left\langle\mathbf{C}_{r, s} S(t, r)^{*} x^{*}, S(t, r)^{*} x^{*}\right\rangle=\left\langle\mathbf{C}_{t, s} x^{*}, x^{*}\right\rangle-\left\langle\mathbf{C}_{t, r} x^{*}, x^{*}\right\rangle \leq\left\langle\mathbf{C}_{t, s} x^{*}, x^{*}\right\rangle=\left|\mathbf{C}_{t, s} x^{*}\right|_{H_{t, s}}^{2} .
$$

Hence,

$$
\left|\left\langle\mathbf{C}_{r, s} S(t, r)^{*} x^{*}, y^{*}\right\rangle\right|=\left|\left[\mathbf{C}_{r, s} S(t, r)^{*} x^{*}, \mathbf{C}_{r, s} y^{*}\right]_{H_{r, s}}\right| \leq\left|\mathbf{C}_{t, s} x^{*}\right|_{H_{t, s}}\left|\mathbf{C}_{r, s} y^{*}\right|_{H_{r, s}} .
$$

For $y^{*} \in \mathbf{E}^{*}$ fixed we define the linear functional $\psi_{y^{*}}$ : Range $\mathbf{C}_{t, s} \rightarrow \mathbb{R}$ by

$$
\psi_{y^{*}}\left(\mathbf{C}_{t, s} x^{*}\right):=\left\langle\mathbf{C}_{r, s} S(t, r)^{*} x^{*}, y^{*}\right\rangle .
$$

This is well-defined since, by (5.6), if $\mathbf{C}_{t, s} x^{*}=0$ then $\mathbf{C}_{r, s} S(t, r)^{*} x^{*}=0$. By (5.7) $\psi_{y^{*}}$ extends to a bounded linear functional on $H_{t, s}$ with norm bounded by $\left|\mathbf{C}_{r, s} y^{*}\right|_{H_{r, s}}$. Identifying $\psi_{y^{*}}$ with an element of $H_{t, s}$, for all $x \in \mathbf{E}^{*}$ we have

$$
\left\langle\psi_{y^{*}}, x^{*}\right\rangle=\left[\mathbf{C}_{t, s} x^{*}, \psi_{y^{*}}\right]_{H_{t, s}}=\left\langle\mathbf{C}_{r, s} S(t, r)^{*} x^{*}, y^{*}\right\rangle=\left\langle S(t, r) \mathbf{C}_{r, s} y^{*}, x^{*}\right\rangle .
$$

Therefore, $S(t, r) \mathbf{C}_{r, s} y^{*}=\psi_{y^{*}} \in H_{t, s}$ and $\left|S(t, r) \mathbf{C}_{r, s} y^{*}\right|_{H_{t, s}} \leq\left|\mathbf{C}_{r, s} y^{*}\right|_{H_{r, s}}$, and the desired result follows.

Next we characterize the equality of the Hilbert spaces $H_{t, r}$ and $H_{t, s}$ in terms of the restriction $S(t, r) \in \mathcal{L}\left(H_{r, s}, H_{t, s}\right)$.

Theorem 5.7 For all $0 \leq s<r<t$ we have $H_{t, s}=H_{t, r}$, as subsets of $\mathbf{E}$, if and only if $\|S(t, r)\|_{\mathcal{L}\left(H_{r, s}, H_{t, s}\right)}<1$.

Proof. We know already that $H_{t, r} \subset H_{t, s}$, so it remains to prove that $H_{t, s} \subset H_{t, r}$, if and only if $\|S(t, r)\|_{\mathcal{L}\left(H_{r, s}, H_{t, s}\right)}<1$.

We assume first that $\|S(t, r)\|_{\mathcal{L}\left(H_{r, s}, H_{t, s}\right)}<1$. By Theorem 5.6, for $y^{*} \in \mathbf{E}^{*}$ we have $S(t, r) \mathbf{C}_{r, s} y^{*} \in H_{t, s}$. Then, if $x^{*} \in \mathbf{E}^{*}$ it follows that

$$
\left[\mathbf{C}_{r, s} S(t, r)^{*} x^{*}, \mathbf{C}_{r, s} y^{*}\right]_{H_{r, s}}=\left\langle S(t, r)^{*} x^{*}, \mathbf{C}_{r, s} y^{*}\right\rangle=\left\langle x^{*}, S(t, r) \mathbf{C}_{r, s} y^{*}\right\rangle=\left[\mathbf{C}_{t, s} x^{*}, S(t, r) \mathbf{C}_{r, s} y^{*}\right]_{H_{t, s}} .
$$


Hence

$$
\begin{aligned}
\left|\mathbf{C}_{r, s} S(t, r)^{*} x^{*}\right|_{H_{r, s}} & =\sup \left\{\left[\mathbf{C}_{r, s} S(t, r)^{*} x^{*}, \mathbf{C}_{r, s} y^{*}\right]_{H_{r, s}}: y^{*} \in \mathbf{E}^{*},\left|\mathbf{C}_{r, s} y^{*}\right|_{H_{r, s}} \leq 1\right\} \\
& =\sup \left\{\left[\mathbf{C}_{t, s} x^{*}, S(t, r) \mathbf{C}_{r, s} y^{*}\right]_{H_{t, s}}: y^{*} \in \mathbf{E}^{*},\left|\mathbf{C}_{r, s} y^{*}\right|_{H_{r, s}} \leq 1\right\} \\
& \leq\|S(t, r)\|_{\mathcal{L}\left(H_{r, s}, H_{t, s}\right)} \cdot\left|\mathbf{C}_{t, s} x^{*}\right|_{H_{t, s}} .
\end{aligned}
$$

Using the last inequality, we get

$$
\begin{aligned}
\left|\mathbf{C}_{t, s} x^{*}\right|_{H_{t, s}}^{2} & =\left|\mathbf{C}_{t, s} x^{*}\right|_{H_{t, s}}^{2}-\left|\mathbf{C}_{r, s} S(t, r)^{*} x^{*}\right|_{H_{r, s}}^{2}+\left|\mathbf{C}_{r, s} S(t, r)^{*} x^{*}\right|_{H_{r, s}}^{2} \\
& =\left\langle\mathbf{C}_{t, s} x^{*}, x^{*}\right\rangle-\left\langle S(t, r) \mathbf{C}_{r, s} S(t, r)^{*} x^{*}, x^{*}\right\rangle+\left|\mathbf{C}_{r, s} S(t, r)^{*} x^{*}\right|_{H_{r, s}}^{2} \\
& \leq\left\langle\mathbf{C}_{t, r} x^{*}, x^{*}\right\rangle+\|S(t, r)\|_{\mathcal{L}\left(H_{r, s}, H_{t, s}\right)}^{2} \cdot\left|\mathbf{C}_{t, s} x^{*}\right|_{H_{t, s}}^{2}
\end{aligned}
$$

That is,

$$
\left\langle\mathbf{C}_{t, s} x^{*}, x^{*}\right\rangle=\left|\mathbf{C}_{t, s} x^{*}\right|_{H_{t, s}}^{2} \leq \frac{1}{1-\|S(t, r)\|_{\mathcal{L}\left(H_{r, s}, H_{t, s}\right)}^{2}}\left\langle\mathbf{C}_{t, r} x^{*}, x^{*}\right\rangle .
$$

By Proposition 2.3, this implies the inclusion $H_{t, s} \subset H_{t, r}$. Conversely, assume that $H_{t, s} \subset H_{t, r}$. Then there exists $K>0$ such that

$$
\left\langle\mathbf{C}_{t, s} x^{*}, x^{*}\right\rangle \leq K\left\langle\mathbf{C}_{t, r} x^{*}, x^{*}\right\rangle=K\left\langle\mathbf{C}_{t, s} x^{*} \cdot x^{*}\right\rangle-K\left\langle S(t, r) \mathbf{C}_{r, s} S(t, r)^{*} x, x^{*}\right\rangle
$$

for all $x^{*} \in \mathbf{E}^{*}$. Notice that $K>1$ since $\left\langle\mathbf{C}_{t, r} x^{*}, x^{*}\right\rangle \leq\left\langle\mathbf{C}_{t, s} x^{*}, x^{*}\right\rangle$ for all $x \in \mathbf{E}^{*}$. Then, the above inequality yields

$$
\left|\mathbf{C}_{r, s} S(t, r)^{*} x\right|_{H_{r, s}}^{2} \leq\left(1-\frac{1}{K}\right)\left|\mathbf{C}_{t, s} x^{*}\right|_{H_{t, s}}^{2}
$$

Using (5.8) again we get

$$
\begin{aligned}
\left|\left[S(t, r) \mathbf{C}_{r, s} y^{*}, \mathbf{C}_{t, s} x^{*}\right]_{H_{t, s}}\right| & =\left|\left[\mathbf{C}_{r, s} y^{*}, \mathbf{C}_{r, s} S(t, r)^{*} x^{*}\right]_{H_{r, s}}\right| \\
& \leq\left|\mathbf{C}_{r, s} y^{*}\right|_{H_{r, s}} \cdot\left|\mathbf{C}_{r, s} S(t, r)^{*} x^{*}\right|_{H_{r, s}} \\
& \leq\left(1-\frac{1}{K}\right)^{1 / 2}\left|\mathbf{C}_{r, s} y^{*}\right|_{H_{r, s}} \cdot\left|\mathbf{C}_{t, s} x^{*}\right|_{H_{t, s}}
\end{aligned}
$$

which shows that $\|S(t, r)\|_{\mathcal{L}\left(H_{r, s}, H_{t, s}\right)} \leq\left(1-\frac{1}{K}\right)^{1 / 2}<1$.

Finally, we establish the smoothing property of the Ornstein-Uhlenbeck transition operators. We need the following assumption, usually referred to as null-controllability condition (see Remark 5.9 below).

Assumption A.2 For all $(t, s) \in \mathfrak{T}$ we have

$$
\text { Range } S(t, s) \subset H_{t, s}
$$

Notation If condition (5.9) holds, we denote by $\Sigma(t, s)$ the map $S(t, s)$ regarded as an operator from $\mathbf{E}$ into $H_{t, s}$. Notice that $\Sigma(t, s)$ is bounded by the Closed-Graph Theorem, and we have $S(t, s)=i_{t, s} \circ \Sigma(t, s)$.

As in (2.3), let $\phi_{t, s}: H_{t, s} \rightarrow L^{2}\left(\mathbf{E}, \mu_{t, s}\right)$ denote the unique bounded extension of the isometry

$$
\mathbf{C}_{t, s}\left(\mathbf{E}^{*}\right) \ni \mathbf{C}_{t, s} x^{*} \mapsto\left\langle x^{*}, \cdot\right\rangle \in L^{2}\left(\mathbf{E}, \mu_{t, s}\right)
$$

Let $\mathbf{C}_{b}^{\infty}(\mathbf{E})$ denote the set of infinitely Fréchet-differentiable real-valued functions on E. Using Proposition 2.6 together with the condition (5.9) we obtain the following

Theorem 5.8 Let Assumptions (AT), A.1 and A.2 be satisfied. Then the Ornstein-Uhlenbeck transition operators $\{P(s, t)\}_{(t, s) \in \mathfrak{T}}$ satisfy

$$
\varphi \in \mathcal{B}_{b}(\mathbf{E}) \Rightarrow P(s, t) \varphi \in C_{b}^{\infty}(\mathbf{E}) .
$$

The Fréchet derivative of the function $P(s, t) \varphi: \mathbf{E} \rightarrow \mathbb{R}$ at $x \in \mathbf{E}$ in the direction $y \in \mathbf{E}$ is given by

$$
\langle D P(s, t) \varphi(x), y\rangle=\int_{\mathbf{E}} \varphi(S(t, s) x+z) \phi_{t, s}(\Sigma(t, s) y)(z) \mu_{t, s}(d z),
$$


and the second Fréchet derivative of $P(s, t) \varphi$ at $x \in \mathbf{E}$ in the directions $y_{1}, y_{2} \in \mathbf{E}$ is given by

$$
\begin{aligned}
& \left\langle D^{2} P(s, t) \varphi(x) y_{1}, y_{2}\right\rangle \\
= & -P(s, t) \varphi(x)\left[\Sigma(t, s) y_{1}, \Sigma(t, s) y_{2}\right]_{H_{t, s}}+\int_{\mathbf{E}} \varphi(S(t, s) x+z) \phi_{t, s}\left(\Sigma(t, s) y_{1}\right)(z) \phi_{t, s}\left(\Sigma(t, s) y_{2}\right)(z) \mu_{t, s}(d z)
\end{aligned}
$$

In particular, we have the estimates

$$
\begin{gathered}
\left\|D_{x} P(s, t) \varphi(x)\right\|_{\mathbf{E}^{*}} \leq\|\Sigma(t, s)\|_{\mathcal{L}\left(\mathbf{E}, H_{t, s}\right)}|\varphi|_{0} \\
\left\|D_{x}^{2} P(s, t) \varphi(x)\right\|_{\mathcal{L}\left(\mathbf{E}, \mathbf{E}^{*}\right)} \leq 2\|\Sigma(t, s)\|_{\mathcal{L}\left(\mathbf{E}, H_{t, s}\right)}^{2}|\varphi|_{0} .
\end{gathered}
$$

Remark 5.9 The condition (5.9) has a well-known control theoretic interpretation: for each $s \in[0, T]$ consider the nonhomogeneous Cauchy problem

$$
\begin{aligned}
y^{\prime}(t)+A(t) y(t) & =G(t) u(t), \quad t \in[s, T], \\
y(s) & =x \in \mathbf{E},
\end{aligned}
$$

with $u \in L^{2}(s, T ; \mathbf{H})$. The mild solution of (5.12) is defined as

$$
y^{x, u}(t):=S(t, s) x+\int_{s}^{t} S(t, r) G(r) u(r) d r, \quad t \in[s, T] .
$$

We say that (5.12) is null-controllable in time $t$ iff for all $x \in \mathbf{E}$ there exists a control $u \in L^{2}(s, t ; \mathbf{H})$ such that $y^{x, u}(t)=0$. Using the following characterization of the Hilbert spaces $H_{t, s}$,

$$
H_{t, s}=\left\{\int_{t}^{s} S(t, r) G(r) u(r) d r: u \in L^{2}(s, t ; \mathbf{H})\right\}, \quad(t, s) \in \mathfrak{T}
$$

(see e.g. van Neerven, 2001, Lemma 5.2) it follows that (5.12) is null-controllable in time $t$ if and only if condition (5.9) holds, and we have

$$
|x|_{H_{t, s}}=\inf \left\{|u|_{L^{2}(s, t ; \mathbf{H})}: u \in L^{2}(s, t ; \mathbf{H}) \text { and } \int_{s}^{t} S(t, r) G(r) u(r) d r=x\right\} .
$$

That is, $|x|_{H_{t, s}}^{2}$ is the minimal energy needed to steer the control system (5.12) from 0 to $x$ in time $t-s$. Example 5.10 Suppose that for each $t \in[0, T]$ the map $G(t)$ is injective and for each $(t, s) \in \mathfrak{T}$ we have

$$
\text { Range } S(t, s) \subset \text { Range } G(t) \text {. }
$$

Suppose also that for each $s \in[0, T]$ we have

$$
\int_{s}^{T}\left|G(t)^{-1} S(t, s)\right|_{\mathcal{L}(\mathbf{E}, \mathbf{H})}^{2} d t<+\infty .
$$

Then Assumption A.2 holds. Indeed, let $x \in \mathbf{E}$ and $0 \leq s<t \leq T$, and define

$$
u(r):=\frac{1}{t-s} G(r)^{-1} S(r, s) x, \quad r \in[s, t]
$$

Then $u \in L^{2}(s, t ; \mathbf{H})$ and we have

$$
\int_{s}^{t} S(t, r) G(r) u(r) d r=\frac{1}{t-s} \int_{s}^{t} S(t, r) S(r, s) x d r=S(t, s) x
$$

that is, $S(t, s) x \in H_{t, s}$ according to (5.14), and Assumption A.2 follows. Moreover, by (5.15), we have

$$
|\Sigma(t, s) x|_{H_{t, s}} \leq \frac{1}{t-s}|x|_{\mathbf{E}}\left(\int_{s}^{t}\left|G(r)^{-1} S(r, s)\right|_{\mathcal{L}(\mathbf{E}, \mathbf{H})}^{2} d r\right)^{1 / 2}, \quad s<t \leq T
$$




\section{Mild Solutions of Hamilton-Jacobi Equations in Banach Spaces}

Let $\{-A(t)\}_{t \in[0, T]}$ be the generator of an evolution family on a Banach space $\mathbf{E}$. Let $\mathbf{H}$ be a separable Hilbert space and let $\{G(t)\}_{t \in[0, T]}$ be a family of (possibly unbounded) linear operators from $\mathbf{H}$ into $\mathbf{E}$. We consider the HamiltonJacobi equation on $\mathbf{E}$

$$
\begin{aligned}
\frac{\partial v}{\partial t}(t, x)+L_{t} v(t, \cdot)(x)+\mathcal{H}\left(t, x, D_{x} v(t, x)\right) & =0, \quad(t, x) \in[0, T] \times \mathbf{E}, \\
v(T, x) & =\varphi(x) .
\end{aligned}
$$

The final condition $\varphi: \mathbf{E} \rightarrow \mathbb{R}$ and the nonlinear Hamiltonian operator $\mathcal{H}:[0, T] \times \mathbf{E} \times \mathbf{E}^{*} \rightarrow \mathbb{R}$ are given, and for each $t \in[0, T], L_{t}$ is the second-order differential operator

$$
\left(L_{t} \phi\right)(x):=-\left\langle A(t) x, D_{x} \phi(x)\right\rangle+\frac{1}{2} \operatorname{Tr}_{\mathbf{H}}\left[G(t)^{*} D_{x}^{2} \phi(x) G(t)\right], \quad x \in D(A(t)), \phi \in C_{b}^{2}(\mathbf{E}) .
$$

Using the associated OU-transition evolution operators $\{P(s, t)\}_{(t, s) \in \mathfrak{T}}$ (see Definition 5.4) we rewrite Equation (6.1) in the integral form

$$
v(t, x)=[P(t, T) \varphi](x)+\int_{t}^{T}\left[P(t, s) \mathcal{H}\left(s, \cdot, D_{x} v(s, \cdot)\right)\right](x) d s .
$$

Observe that the trace term in (6.1) may not be well-defined since $G(t)$ is not necessarily a bounded operator.

Definition 6.1 For $\alpha \in(0,1)$, we denote with $\mathfrak{S}_{T, \alpha}$ the set of bounded and measurable functions $v:[0, T] \times \mathbf{E} \rightarrow \mathbb{R}$ such that $v(t, \cdot) \in C_{b}^{1}(\mathbf{E})$, for all $t \in[0, T)$, and the mapping

$$
[0, T) \times \mathbf{E} \ni(t, x) \mapsto(T-t)^{\alpha} D_{x} v(t, x) \in \mathbf{E}^{*}
$$

is bounded and measurable.

The space $\mathfrak{S}_{T, \alpha}$ is a Banach space endowed with the norm

$$
\|v\|_{\mathfrak{S}_{T, \alpha}}:=\sup _{t \in[0, T]}\|v(t, \cdot)\|_{0}+\sup _{t \in[0, T]}(T-t)^{\alpha}\left\|D_{x} v(t, \cdot)\right\|_{0} .
$$

Definition 6.2 We will say that a function $v:[0, T] \times \mathbf{E} \rightarrow \mathbb{R}$ is a mild solution of the Hamilton-Jacobi Equation (6.1) if $v \in \mathfrak{S}_{T, \alpha}$ for some $\alpha \in(0,1)$, for each $(t, x) \in[0, T] \times \mathbf{E}$ the mapping

$$
[t, T] \ni s \mapsto\left[P(t, s) \mathcal{H}\left(s, \cdot, D_{x} v(s, \cdot)\right)\right](x) \in \mathbb{R}
$$

is integrable and $v$ satisfies (6.2).

Assumption A.3 There exists $\alpha \in(0,1)$ and $C>0$ such that

$$
|\Sigma(t, s)|_{\mathcal{L}\left(\mathbf{E}, H_{t, s}\right)} \leq C(t-s)^{-\alpha}, \quad 0 \leq s<t \leq T .
$$

Example 6.3 Under the same assumptions of Example 5.10, assume further that there exists $\beta \in\left[0, \frac{1}{2}\right)$ and $C>0$ such that

$$
\left|G(t)^{-1} S(t, s)\right|_{\mathcal{L}(\mathbf{E}, \mathbf{H})} \leq C(t-s)^{-\beta}, \quad 0 \leq s<t \leq T .
$$

Then Assumption A.3 holds with $\alpha=\beta+\frac{1}{2}$.

Assumption A.4 For all $(t, p) \in[0, T] \times \mathbf{E}^{*}$, the map

$$
\mathbf{E} \ni x \mapsto \mathcal{H}(t, x, p) \in \mathbb{R}
$$

is continuous and bounded, and there exists $C>0$ such that

$$
|\mathcal{H}(t, x, p)-\mathcal{H}(t, x, q)| \leq C|p-q|_{\mathbf{E}^{*}}, \quad t \in[0, T], \quad x \in \mathbf{E}, \quad p, q \in \mathbf{E}^{*} .
$$

Example 6.4 If the Hamiltonian $\mathcal{H}$ has the form

$$
\mathcal{H}(t, x, p)=\inf _{u \in M}\{\langle F(t, x, u), p\rangle+l(t, x, u)\}, \quad(t, x, p) \in[0, T] \times \mathbf{E} \times \mathbf{E}^{*}
$$


where $M$ is a separable metric space, $F:[0, T] \times \mathbf{E} \times M \rightarrow \mathbf{E}$ is uniformly bounded and weakly-continuous in $x \in \mathbf{E}$ uniformly with respect to $u \in M$, and $l:[0, T] \times \mathbf{E} \times M \rightarrow(-\infty, \infty]$ is continuous in $x \in \mathbf{E}$ uniformly with respect to $u \in M$ and satisfies

$$
\sup _{x \in \mathbf{E}, u \in M}|l(t, x, u)|<+\infty, \forall t \in[0, T]
$$

then Assumption A.4 holds, see e.g. the proof of Theorem 10.1 in (Fleming \& Soner, 2006, Chapter II) or (Bardi \& Capuzzo-Dolcetta, 1997, Chapter III, Lemma 2.11).

Recall that if Hamiltonian $\mathcal{H}$ takes the form (6.3), Equation (6.1) is the Hamilton-Jacobi-Bellman PDE associated with the dynamic programming approach to stochastic optimal control problems of the form

$$
\operatorname{minimize} \quad J(U)=\mathbb{E}\left[\int_{0}^{T} l\left(t, X^{U}(t), U(t)\right) d t+\Psi(X(T))\right]
$$

subject to

- $U=\{U(t)\}_{t \in[0, T]}$ is an $M$-valued control process

- $\left\{X^{U}(t)\right\}_{t \in[0, T]}$ is the E-valued solution to the controlled non-autonomous stochastic evolution equation with additive noise

$$
\begin{aligned}
d X(t)+A(t) X(t) d t & =F(t, X(t), u(t)) d t+G(t) d W(t) \\
X(0) & =x_{0} \in \mathbf{E} .
\end{aligned}
$$

Remark 6.5 A Banach-space framework seems more suitable for certain control problems for stochastic PDEs. Consider for instance the following controlled stochastic PDE of reaction-diffusion type perturbed by additive space-time white noise on $[0, T] \times(0,1)$,

$$
\begin{aligned}
\frac{\partial X}{\partial t}(t, \xi)+\left(\mathcal{A}_{t} X\right)(t, \xi) & =f(X(t, \xi), U(t))+g(t, \xi) \frac{\partial w}{\partial t}(t, \xi), \\
X(t, 0)=X(t, 1) & =0 \\
X(0, \cdot) & =x_{0}(\cdot)
\end{aligned}
$$

where, for each $t \in[0, T], \mathcal{A}_{t}$ denotes the second order differential operator introduced in Example 5.3. In applications, it is useful to study running cost functions that allow to regulate the solution $X(\cdot)$ at some fixed points $\zeta_{1}, \ldots, \zeta_{n} \in(0,1)$, say

$$
J(U)=\mathbb{E}\left[\int_{0}^{T} \phi\left(t, X^{U}\left(t, \zeta_{1}\right), \ldots, X^{U}\left(t, \zeta_{n}\right), U(t)\right) d t\right] .
$$

This running cost functional clearly requires that the solution $X(t, \xi)$ is continuous with respect to the space variable $\xi$. Recently, Veraar (2010) (see also Veraar \& Zimmerschied, 2008) have proved that weak solutions to the uncontrolled version of Equation (6.5) exist and have trajectories almost surely in

$$
C\left([0, T] ; D\left(A_{p}(0)^{\delta}\right)\right) \text { for } \delta<\frac{1}{4}
$$

where $A_{p}(t)$ denotes the realization in $L^{p}(0,1)$ of $\mathcal{A}_{t}$ with zero-Dirichlet boundary conditions, see Example 5.3. If we choose

$$
p>2 \text { and } \delta \in\left(\frac{1}{2 p}, \frac{1}{4}\right)
$$

using Theorem 1.15.3 in Triebel (1978) and Sobolev's embedding theorem, it follows

$$
D\left(A_{p}(0)^{\delta}\right)=\left[L^{p}(0,1), D\left(A_{p}(0)\right)\right]_{\delta}=H_{0}^{2 \delta, p}(0,1) \hookrightarrow C_{0}[0,1]
$$

that is, cost functional (6.6) is now well-defined. This suggests to choose $\mathbf{E}=L^{p}(0,1)$ with $p>2$ as state space for the above control problem and the associated Hamilton-Jacobi-Bellman Equation (6.1).

As we mentioned, at the moment we are unable to obtain optimality criteria and verification-type results for optimal control problems in Banach spaces for non-autonomous stochastic PDEs as this requires approximation results of in $C_{b}(\mathbf{E})$ by smooth functions that do not seem available at the moment in the general Banach-space setting. We will address this issue in a forthcoming paper. 
We now present the final result of this paper, which generalizes to the non-autonomous Banach-space setting Theorem 9.3 in Zabczyk (1999) on existence of mild solutions to HJ equations in Hilbert spaces (see also Da Prato \& Zabczyk, 2002, Part III and Masiero, 2005).

Theorem 6.6 Let $\varphi \in \mathcal{C}_{b}(\mathbf{E})$. Suppose Assumptions (AT) and A.1-A.4 hold true. Then there exists a unique mild solution to Equation (6.1).

Proof. The argument is largely based on the proof of Theorem 2.9 in Masiero (2005). For any $v \in \mathfrak{S}_{T, \alpha}$ we define the function $\gamma(v)$ by

$$
\gamma(v)(t, x):=[P(t, T) \varphi](x)+\int_{t}^{T}\left[P(t, s) \mathcal{H}\left(s, \cdot, D_{x} v(s, \cdot)\right)\right](x) d s,
$$

for $(t, x) \in[0, T] \times \mathbf{E}$. By Theorem 5.8, estimate (5.10) and Assumptions A.3-A.4, the map $\gamma(v)$ belongs to $\mathfrak{S}_{T, \alpha}$. We will show that $\gamma$ is a strict contraction on $\mathfrak{S}_{T, \alpha}$ when endowed with the equivalent norm

$$
\|v\|_{\beta, \mathfrak{S}_{T, \alpha}}:=\sup _{t \in[0, T]} e^{-\beta(T-t)}\left[\|v(t, \cdot)\|_{0}+(T-t)^{\alpha}\left\|D_{x} v(t, \cdot)\right\|_{0}\right]
$$

and $\beta>0$ is a parameter to be specified below. Let $v_{1}, v_{2} \in \mathfrak{S}_{T, \alpha}$.

STEP 1. From Assumptions A.3-A.4 and estimate (5.10), we obtain

$$
\begin{aligned}
\left|\gamma\left(v_{1}\right)(t, x)-\gamma\left(v_{2}\right)(t, x)\right| & \leq \int_{t}^{T}\left|\left[P(t, s)\left(\mathcal{H}\left(s, \cdot, D_{x} v_{1}(s, \cdot)\right)-\mathcal{H}\left(s, \cdot, D_{x} v_{2}(s, \cdot)\right)\right)\right](x)\right| d s \\
& \leq C \int_{t}^{T}\left|D_{x} v_{1}(s, x)-D_{x} v_{2}(s, x)\right|_{\mathbf{E}^{*}} d s \\
& \leq C \int_{t}^{T}(T-s)^{-\alpha} e^{\beta(T-s)}\left\|v_{1}-v_{2}\right\|_{\beta, \mathfrak{S}_{T, \alpha}} d s .
\end{aligned}
$$

Let $\varepsilon \in(0,1)$. We can estimate the above integral as follows

$$
\begin{aligned}
\int_{t}^{T}(T-s)^{-\alpha} e^{\beta(T-s)} d s & =(T-t)^{1-\alpha} \int_{0}^{1} r^{-\alpha} e^{\beta(T-t) r} d r \\
& =(T-t)^{1-\alpha}\left[\int_{0}^{\varepsilon} r^{-\alpha} e^{\beta(T-r)} d r+\int_{\varepsilon}^{1} r^{-\alpha} e^{\beta(T-t) r} d r\right] \\
& \leq \frac{(T-t)^{1-\alpha}}{1-\alpha}\left[\varepsilon^{1-\alpha} e^{\beta(T-t) \varepsilon}+\left(1-\varepsilon^{1-\alpha}\right) e^{\beta(T-t)}\right]
\end{aligned}
$$

Then

$$
\begin{aligned}
& e^{-\beta(T-t)}\left\|\gamma\left(v_{1}\right)(t, \cdot)-\gamma\left(v_{2}\right)(t, \cdot)\right\|_{0} \\
\leq & \frac{C(T-t)^{1-\alpha}}{1-\alpha}\left[\varepsilon^{1-\alpha} e^{-\beta(T-t)(1-\varepsilon)}+\left(1-\varepsilon^{1-\alpha}\right)\right]\left\|v_{1}-v_{2}\right\|_{\beta, \mathfrak{S}_{T, \alpha}} .
\end{aligned}
$$

We may choose $\varepsilon_{1} \in(0,1)$ such that

$$
\frac{C T^{1-\alpha}}{1-\alpha}\left(1-\varepsilon_{1}^{1-\alpha}\right)<\frac{1}{5}
$$

Now, for $\beta>\frac{1-\alpha}{T\left(1-\varepsilon_{1}\right)}$ the map

$$
[0, T] \ni t \mapsto\left[\varepsilon_{1}(T-t)\right]^{1-\alpha} e^{-\beta(T-t)\left(1-\varepsilon_{1}\right)}
$$

attains its global maximum at $\bar{t}=T-\frac{1-\alpha}{\beta\left(1-\varepsilon_{1}\right)}$. Hence,

$$
\sup _{t \in[0, T]}\left[\varepsilon_{1}(T-t)\right]^{1-\alpha} e^{-\beta(T-t)\left(1-\varepsilon_{1}\right)}=\left[\frac{\varepsilon_{1}(1-\alpha)}{e \beta\left(1-\varepsilon_{1}\right)}\right]^{1-\alpha}
$$

which tends to zero as $\beta \rightarrow \infty$. Therefore, we can choose $\beta_{1}=\beta_{1}\left(\varepsilon_{1}\right)$ sufficiently large such that

$$
\sup _{t \in[0, T]} \frac{C\left[\varepsilon_{1}(T-t)\right]^{1-\alpha}}{1-\alpha} e^{-\beta_{1}(T-t)\left(1-\varepsilon_{1}\right)}<\frac{1}{5} .
$$


Thus, if $\varepsilon \in\left(\varepsilon_{1}, 1\right)$ and $\beta>\beta_{1}(\varepsilon)$, we have

$$
\sup _{t \in[0, T]} e^{-\beta(T-t)}\left\|\gamma\left(v_{1}\right)(t, \cdot)-\gamma\left(v_{2}\right)(t, \cdot)\right\|_{0} \leq \frac{2}{5}\left\|v_{1}-v_{2}\right\|_{\beta, \mathfrak{S}_{T, \alpha}} .
$$

SteP 2. Using again Assumption A.3 and estimate (5.10), it follows that

$$
\begin{aligned}
\left|D_{x} \gamma\left(v_{1}\right)(t, x)-D_{x} \gamma\left(v_{2}\right)(t, x)\right|_{\mathbf{E}^{*}} & \leq \int_{t}^{T}\left|D_{x} P(t, s)\left[\mathcal{H}\left(s, \cdot, D_{x} v_{1}(s, \cdot)\right)-\mathcal{H}\left(s, \cdot, D_{x} v_{2}(s, \cdot)\right)\right](x)\right|_{\mathbf{E}^{*}} d s \\
& \leq C \int_{t}^{T}(s-t)^{-\alpha}\left|\mathcal{H}\left(s, \cdot, D_{x} v_{1}(s, \cdot)\right)-\mathcal{H}\left(s, \cdot, D_{x} v_{2}(s, \cdot)\right)\right| d s \\
& \leq C^{2} \int_{t}^{T}(s-t)^{-\alpha}\left|D_{x} v_{1}(s, x)-D_{x} v_{2}(s, x)\right|_{\mathbf{E}^{*}} d s \\
& \leq C^{2} \int_{t}^{T}(s-t)^{-\alpha}(T-s)^{-\alpha} e^{\beta(T-s)}\left\|v_{1}-v_{2}\right\|_{\beta, \mathfrak{S}_{T, \alpha}} d s
\end{aligned}
$$

For the last integral we have

$$
\begin{aligned}
& \int_{t}^{T}(s-t)^{-\alpha}(T-s)^{-\alpha} e^{\beta(T-s)} d s \\
= & (T-t)^{1-2 \alpha}\left[\int_{0}^{\varepsilon}(1-r)^{-\alpha} r^{-\alpha} e^{\beta(T-t) r} d r+\int_{\varepsilon}^{1}(1-r)^{-\alpha} r^{-\alpha} e^{\beta(T-t) r} d r\right] \\
\leq & \frac{(T-t)^{1-2 \alpha}}{1-\alpha}\left[(1-\varepsilon)^{-\alpha} \varepsilon^{1-\alpha} \exp (\beta(T-t) \varepsilon)+(1-\varepsilon)^{1-\alpha} \varepsilon^{-\alpha} \exp (\beta(T-t))\right] .
\end{aligned}
$$

Hence,

$$
\begin{aligned}
& e^{\beta(T-t)}(T-t)^{\alpha}\left\|D_{x} \gamma\left(v_{1}\right)(t, \cdot)-D_{x} \gamma\left(v_{2}\right)(t, \cdot)\right\|_{0} \\
\leq & \frac{C^{2}(T-t)^{1-\alpha}}{1-\alpha}\left[(1-\varepsilon)^{-\alpha} \varepsilon^{1-\alpha} \exp \left(\beta(T-t)(\varepsilon-1)+(1-\varepsilon)^{1-\alpha} \varepsilon^{-\alpha}\right]\left\|v_{1}-v_{2}\right\|_{\beta, \mathfrak{S}_{T, \alpha}} .\right.
\end{aligned}
$$

As in step 1 , we may choose $\varepsilon_{2} \in(0,1)$ such that

$$
\frac{C^{2} \varepsilon_{2}^{-\alpha}\left[T\left(1-\varepsilon_{2}\right)\right]^{1-\alpha}}{1-\alpha}<\frac{1}{5}
$$

and $\beta_{2}=\beta_{2}\left(\varepsilon_{2}\right)>0$ sufficiently large such that

$$
\sup _{t \in[0, T]} \frac{C^{2}\left[\varepsilon_{2}(T-t)\right]^{1-\alpha}\left(1-\varepsilon_{2}\right)^{-\alpha}}{1-\alpha} \exp \left(\beta_{2}(T-t)\left(\varepsilon_{2}-1\right)<\frac{1}{5} .\right.
$$

Thus, for $\varepsilon \in\left(\varepsilon_{2}, 1\right)$ and $\beta>\beta_{2}(\varepsilon)$,

$$
\sup _{t \in[0, T]} e^{-\beta(T-t)}(T-t)^{\alpha}\left\|D_{x} \gamma\left(v_{1}\right)(t, \cdot)-D_{x} \gamma\left(v_{2}\right)(t, \cdot)\right\|_{0} \leq \frac{2}{5}\left\|v_{1}-v_{2}\right\|_{\beta, \mathfrak{S}_{T, \alpha}} .
$$

We conclude that for $\varepsilon \in\left(\max \left\{\varepsilon_{1}, \varepsilon_{2}\right\}, 1\right)$ and $\beta>\max \left\{\beta_{1}(\varepsilon), \beta_{2}(\varepsilon)\right\}$ we have

$$
\left\|\gamma\left(v_{1}\right)-\gamma\left(v_{2}\right)\right\|_{\beta, \mathfrak{S}_{T, \alpha}} \leq \frac{4}{5}\left\|\gamma\left(v_{1}\right)-\gamma\left(v_{2}\right)\right\|_{\beta, \mathfrak{S}_{T, \alpha}}
$$

and the desired result follows from the Banach fixed point Theorem.

Example 6.7 Let $p \geq 2$ be fixed and let $A_{p}(t)$ and $G(t)$ be as in Example 5.3, with $g$ satisfying $k_{1}<|g(t, \xi)|<k_{2}$ for all $(t, \xi) \in[0, T] \times(0,1)$, for some $k_{1}>0$. Let $p^{*}:=p /(p-1)$ and let $\mathcal{H}:[0, T] \times L^{p}(0,1) \times L^{p^{*}}(0,1) \rightarrow \mathbb{R}$ and $\varphi$ : $L^{p}(0,1) \rightarrow \mathbb{R}$ satisfy Assumption A.4. Consider the following non-autonomous HJ equation on $[0, T] \times L^{p}(0,1)$,

$$
\begin{aligned}
\frac{\partial v}{\partial t}(t, x)+L_{t} v(t, \cdot)(x)+\mathcal{H}\left(t, x, D_{x} v(t, x)\right) & =0, \quad(t, x) \in[0, T] \times L^{p}(0,1), \\
v(T, x) & =\varphi(x),
\end{aligned}
$$

where, for each $t \in[0, T], L_{t}$ denotes the second-order differential operator on $L^{p}(0,1)$,

$$
\left(L_{t} \phi\right)(x):=-\left\langle A_{p}(t) x, D_{x} \phi(x)\right\rangle+\frac{1}{2} \operatorname{Tr}_{L^{2}(0,1)}\left[G(t)^{*} D_{x}^{2} \phi(x) G(t)\right], x \in D\left(A_{p}(t)\right), \phi \in C_{b}^{2}\left(L^{p}(0,1)\right) .
$$


Here $\langle\cdot, \cdot\rangle$ denotes the duality pairing between $L^{p}(0,1)$ and $L^{p^{*}}(0,1)$. As shown in Examples 5.3, 5.10 and 6.3, Assumptions A.1-A.3 also hold. Then, by Theorem 6.6, there exists a unique mild solution to HJ Equation (6.7).

\section{Acknowledgements}

The author thanks Zdzisław Brzeźniak for suggesting using Theorem 3 in (Aronszajn, 1976, Ch 2, Section 1) in the proof of Proposition 2.6.

\section{References}

Acquistapace, P., \& Terreni, B. (1987). A unified approach to abstract linear nonautonomous parabolic equations. Rend. Sem. Mat. Univ. Padova, 78, 47-107.

Amann, H. (1995). Linear and quasilinear parabolic problems. Monographs in Mathematics (Vol. I, Vol. 89). Boston, MA: Birkhäuser Boston Inc. Abstract linear theory.

Aronszajn, N. (1976). Differentiability of lipschitzian mappings between banach spaces. Studia Mathematica 57(2), 147-190.

Bardi, M., \& Capuzzo-Dolcetta, I. (n.d.). Optimal control and viscosity solutions of Hamilton-Jacobi-Bellman equations. Systems \& Control: Foundations \& Applications. http://dx.doi.org/10.1007/978-0-8176-4755-1

Bogachev, V. I. (1998). Gaussian measures. Mathematical Surveys and Monographs (Vol. 62). Providence, RI: American Mathematical Society.

Brzeźniak, Z. (1996). On sobolev and besov spaces regularity of brownian paths. Stochastics and Stochastic Reports, 56(1-2), 1-15. http://dx.doi.org/10.1080/17442509608834032

Cannarsa, P., \& Da Prato, G. (1991). Second-order hamiltonjacobi equations in infinite dimensions. SIAM Journal on Control and Optimization, 29(2), 474-492. http://epubs.siam.org/doi/abs/10.1137/0329026

Cerrai, S. (2001a). Optimal control problems for stochastic reaction-diffusion systems with non-Lipschitz coefficients. SIAM J. Control Optim., 39(6), 1779-1816.

Cerrai, S. (2001b). Second order PDE's in finite and infinite dimension. Lecture Notes in Mathematics (Vol. 1762). Berlin: Springer-Verlag. A probabilistic approach.

Da Prato, G. (1985). Some results on bellman equation in Hilbert spaces. SIAM Journal on Control and Optimization, 23(1), 61C71. http://epubs.siam.org/doi/abs/10.1137/0323006

Da Prato, G., \& Zabczyk, J. (2002). Second order partial differential equations in Hilbert spaces. London Mathematical Society Lecture Note Series (Vol. 293). Cambridge: Cambridge University Press.

Denk, R., Dore, G., Hieber, M., Prüss, J., \& Venni, A. (2004). New thoughts on old results of R. T. Seeley. Math. Ann., 328(4), 545-583. http://dx.doi.org/10.1007/s00208-003-0493-y

Fleming, W. H., \& Soner, H. M. (2006). Controlled Markov processes and viscosity solutions. Stochastic Modelling and Applied Probability (2nd ed., Vol. 25). New York: Springer.

Fuhrman, M., \& Tessitore, G. (2002a). The Bismut-Elworthy formula for backward SDEs and applications to nonlinear Kolmogorov equations and control in infinite dimensional spaces. Stoch. Stoch. Rep., 74(1-2), 429-464.

Fuhrman, M., \& Tessitore, G. (2002b). Nonlinear Kolmogorov equations in infinite dimensional spaces: The backward stochastic differential equations approach and applications to optimal control. Ann. Probab., 30(3), 1397-1465.

Fuhrman, M., \& Tessitore, G. (2004a). Existence of optimal stochastic controls and global solutions of forwardbackward stochastic differential equations. SIAM J. Control Optim., 43(3), 813-830 (electronic).

Fuhrman, M., \& Tessitore, G. (2004b). Infinite horizon backward stochastic differential equations and elliptic equations in Hilbert spaces. Ann. Probab., 32(1B), 607-660.

Gozzi, F. (1995). Regularity of solutions of a second order Hamilton-Jacobi equation and application to a control problem. Comm. Partial Differential Equations, 20(5-6), 775-826.

Gozzi, F. (1996). Global regular solutions of second order Hamilton-Jacobi equations in Hilbert spaces with locally Lipschitz nonlinearities. J. Math. Anal. Appl., 198(2), 399-443. 
Gross, L. (1967). Potential theory on Hilbert space. J. Functional Analysis, 1, 123-181. http://dx.doi.org/10.1016/00221236(67)90030-4

Maas, J. (2010). Malliavin calculus and decoupling inequalities in banach spaces. Journal of Mathematical Analysis and Applications, 363(2), 383-398.

Masiero, F. (2005). Semilinear Kolmogorov equations and applications to stochastic optimal control. Appl. Math. Optim., 51(2), 201-250.

Masiero, F. (2008). Stochastic optimal control problems and parabolic equations in Banach spaces. SIAM J. Control Optim., 47(1), 251-300.

Pazy, A. (1983). Semigroups of linear operators and applications to partial differential equations. Vol. 44 of Applied Mathematical Sciences. New York: Springer-Verlag.

Prüss, J., \& Sohr, H. (1993). Imaginary powers of elliptic second order differential operators in Lp-spaces. $H i$ roshima Math. J., 23(1), 161-192.

Tanabe, H. (1979). Equations of evolution. Vol. 6 of Monographs and Studies in Mathematics, Pitman (Advanced Publishing Program), Boston, Mass. Translated from the Japanese by N. Mugibayashi and H. Haneda.

Triebel, H. (1978). Interpolation theory, function spaces, differential operators. North-Holland Mathematical Library (Vol. 18). Amsterdam: North-Holland Publishing Co.

van Neerven, J. M. A. M. (1998). Nonsymmetric Ornstein-Uhlenbeck semigroups in Banach spaces. J. Funct. Anal., 155(2), 495-535.

van Neerven, J. M. A. M. (2001). Uniqueness of invariant measures for the stochastic Cauchy problem in Banach spaces, in Recent advances in operator theory and related topics (Szeged, 1999). Oper. Theory Adv. Appl. (Vol. 127, pp. 491C517). Birkhäuser, Basel.

van Neerven, J. M. A. M. (2008). Stochastic evolution equations. ISEM Lecture Notes.

van Neerven, J. M. A. M., \& Weis, L. (2005a). Stochastic integration of functions with values in a Banach space. Studia Math., 166(2), 131-170.

van Neerven, J. M. A. M., \& Weis, L. (2005b). Weak limits and integrals of Gaussian covariances in Banach spaces. Probab. Math. Statist., 25 (1, Acta Univ. Wratislav. No. 2784).

Veraar, M. C. (2010). Non-autonomous stochastic evolution equations and applications to stochastic partial differential equations. J. Evol. Equ., 10(1), 85-127. http://dx.doi.org/10.1007/s00028-009-0041-7

Veraar, M., \& Zimmerschied, J. (2008). Non-autonomous stochastic Cauchy problems in Banach spaces. Studia Math., 185(1), 1-34. http://dx.doi.org/10.4064/sm185-1-1

Yagi, A. (1991). Abstract quasilinear evolution equations of parabolic type in Banach spaces. Boll. Un. Mat. Ital. $B$ (7), 5(2), 341-368.

Zabczyk, J. (1999). Parabolic equations on Hilbert spaces, in Stochastic PDE's and Kolmogorov equations in infinite dimensions (Cetraro, 1998), Vol. 1715 of Lecture Notes in Math. (pp. 117C213). Berlin: Springer.

\section{Copyrights}

Copyright for this article is retained by the author(s), with first publication rights granted to the journal.

This is an open-access article distributed under the terms and conditions of the Creative Commons Attribution license (http://creativecommons.org/licenses/by/3.0/). 